\title{
Dynamical and Surface Impacts of the January 2021 Sudden Stratospheric Warming in Novel Aeolus Wind Observations, MLS and ERA5
}

Corwin J. Wright ${ }^{1}$, Richard J. Hall ${ }^{2}$, Timothy P. Banyard ${ }^{1}$, Neil P. Hindley ${ }^{1}$, Daniel M. Mitchell ${ }^{2}$, and

William J. M. Seviour ${ }^{3,4}$

${ }^{1}$ Centre for Space, Atmospheric and Oceanic Science, University of Bath, UK

${ }^{2}$ School of Geographical Sciences and Cabot Institute for the Environment, University of Bristol, UK

${ }^{3}$ College of Engineering, Mathematics and Physical Sciences, University of Exeter, UK

${ }^{4}$ Global Systems Institute, University of Exeter, UK

Correspondence: Corwin Wright (c.wright@bath.ac.uk)

\begin{abstract}
.
Major sudden stratospheric warmings (SSWs) are extreme dynamical events where the usual strong westerly winds of the stratospheric polar vortex temporarily weaken or reverse and polar stratospheric temperatures rise by tens of Kelvin over just a few days. Via dynamical modification of the atmosphere below them, SSWs are believed to be a key contributor to extreme winter weather events at the surface over the following weeks. Due to the major technical challenges involved in measuring wind from space, SSW-induced changes to the structure of the polar vortex have never previously been directly observed at the global scale. Here, we exploit novel observations from ESA's flagship Aeolus wind-profiler mission, supported by additional temperature and geopotential height data from NASA's MLS limb sounder and the ERA5 reanalysis. This allows us to directly examine wind and related dynamical changes associated with the January 2021 major SSW, the first such event in the Aeolus data record. Aeolus is the first satellite mission to systematically and directly acquire profiles of wind, and therefore our results represent the first direct measurements of SSW-induced wind changes at the global scale. We see a complete reversal of the zonal winds in the lower-middle stratosphere, with reversed winds in some geographic regions reaching down to the bottom $2 \mathrm{~km}$ of the atmosphere. These altered winds are associated with major changes to surface temperature patterns, and in particular we see a strong potential linkage from the SSW to extreme winter-weather outbreaks in Greece and Texas during late January and early February. Our results 1) demonstrate the benefits of wind-profiling satellites such as Aeolus in terms of both their direct measurement capability and use in supporting reanalysis-driven interpretation of stratosphere-troposphere coupling signatures, 2) provide a detailed dynamical description of a major weather event, and 3) have implications for the development of Earth-System models capable of accurately forecasting extreme winter weather.
\end{abstract}

\section{Introduction}

Sudden stratospheric warmings (SSWs) are some of the most dramatic dynamical events in the entire atmospheric system. Over just a few days, temperatures in the winter polar stratosphere can rise by as much as $50 \mathrm{~K}$, while the circumpolar wind jet 
https://doi.org/10.5194/wcd-2021-16

Preprint. Discussion started: 30 March 2021

(c) Author(s) 2021. CC BY 4.0 License.

Weather and

Climate Dynamics

Discussions

L

which usually separates the cold polar stratospheric vortex from the wider atmosphere dramatically reduces in speed and, at many heights and locations, can even reverse. These dynamical changes have major effects on both weather and longer-scale atmospheric processes: in addition to direct local changes to polar ozone and other chemistry (Tao et al., 2015; Safieddine et al., 2020), the altered wind patterns couple outwards to the broader atmospheric system with significant and widespread ramifications (Pedatella et al., 2018). In particular, SSWs often act as the trigger for extreme winter weather events at ground level in the heavily-populated midlatitude regions of North America and Europe, with significant social, safety and economic impacts (Kretschmer et al., 2018; Charlton-Perez et al., 2020; Hall et al., 2020).

The collapse of the circumpolar wind jet is arguably the most important factor affecting how SSWs interact with the broader 30 Earth system. Reflecting this importance, the most broadly-used definition of a major SSW is wind- rather than temperaturebased: specifically, a major SSW is usually defined as a wintertime event in which the zonal mean at $60^{\circ}$ and $10 \mathrm{hPa}$ reaches zero (Charlton and Polvani, 2007). However, direct observations of the vortex winds are extremely technically challenging to make. While wind speed measurements using in-situ radiosondes and ground-based remote-sensing techniques such as lidar and radar are routinely made at many sites, the point-based nature of these techniques inherently limits their ability to characterise the large-scale changes SSWs induce in wind patterns.

Due to these observational limitations, the vast majority of recent research on how SSWs affect winds above the very bottom of the atmosphere has been carried out using models, reanalyses and winds inferred from gradients in other measured variables such as temperature. While these tools have proven highly effective and have significantly advanced our ability to predict winter weather around SSW events, they are not true measurements of wind speed — instead, they infer the wind state through a combination of numerical computation and/or how the wind affects and is affected by other atmospheric parameters.

Here, we exploit data from the European Space Agency (ESA)'s flagship Aeolus satellite mission to directly measure SSW effects on winds in the lower-stratospheric vortex for the first time. Launched in mid-2018, Aeolus is the first satellite instrument capable of systematically and directly measuring winds in the global free troposphere and lower stratosphere, and as such provides a unique window on how SSWs affect winds throughout the lower and lower-middle atmosphere. While Aeolus does not routinely measure winds as high as the $10 \mathrm{hPa}(\sim 32 \mathrm{~km})$ level typically used to diagnose the presence of a major SSW, measurements are available from the surface to $\sim 24 \mathrm{~km}(30 \mathrm{hPa})$. This height range allows us to study both a large fraction of the direct polar vortical changes induced by SSWs and also examine how these changes affect winds at all heights below.

We support and contextualise these Aeolus data with temperature observations and geostrophic wind estimates inferred from the Microwave Limb Sounder on NASA's Aura satellite and with wind, temperature and geopotential height (GPH) output from the European Centre for Medium-Range Weather Forecasts' ERA5 reanalysis. We also investigate the potential surface impacts of the SSW, using temperature and GPH data from ERA5 and snow cover extent data from NOAA. We describe the Aeolus, MLS and ERA5 data in detail in Section 2; the snow cover extent data are only used briefly in this study and are described more fully by Robinson et al. (2014). In addition, Appendix A describes and justifies the geometric adjustments we make to the Aeolus observations to produce the projected zonal and meridional wind products we use throughout this study. 
We use these data to study the major SSW of early January 2021. This was the first and, to date, only SSW to occur during standard Aeolus operations ${ }^{1}$. Hence, we have designed our study to address the twin objectives of (1) characterising SSWinduced dynamical changes for this event using novel observational data and (2) assessing the suitability of Aeolus data for studying future extreme weather events of this type. We address these objectives in parallel throughout the study, rather than taking a series approach, in order to provide a clear narrative based around the physics of the event.

We first place the January $2021 \mathrm{SSW}$ in its broader climatological context in Section 3, using ERA5 and MLS data for all winters since MLS launched in 2004. Section 4 then describes the evolution of the 2021 event specifically using zonal-mean Aeolus and MLS observations. We follow this with a brief discussion of the nature of the event in terms of vortex summary metrics in Section 5, and a more detailed analysis of mesoscale eddy forcing around the SSW period in Section 6. We then examine structural changes to the vortex at a sub-zonal level in Section 7, both in terms of Aeolus wind measurements (Section 7.1) and ERA5 GPH output (Section 7.2). Finally, in Section 8 we study possible surface impacts of the event, before discussing our results and drawing conclusions in Section 9.

\section{Data}

\subsection{Aeolus}

Aeolus is ESA's fifth Earth Explorer mission (Stoffelen et al., 2005; ESA, 2008; Reitebuch, 2012), which launched in mid-2018.

The Aeolus satellite itself is a polar orbiter, in a $320 \mathrm{~km}$ sun-synchronous dawn-dusk $97^{\circ}$ inclination orbit with equator-crossing local solar times of 06:00 and 18:00 in the descending and ascending node respectively. This orbit approaches each pole 15.6 times per day, and thus provides a platform well-suited to characterising polar atmospheric dynamics.

The satellite carries a single primary payload, the Atmospheric Laser Doppler Instrument (ALADIN; Chanin et al., 1989; ESA, 1989). This is a Doppler wind lidar designed to measure winds, aerosol and cloud in the bottom $30 \mathrm{~km}$ of the atmosphere. A $335 \mathrm{~nm}$ laser directed at $35^{\circ}$ off-nadir and $90^{\circ}$ off-track measures both molecular (Rayleigh) and aerosol (Mie) backscatter. We use Rayleigh data, which has a vertical resolution $\sim 0.5-1 \mathrm{~km}$ depending on scanning mode. Following corrections for hot pixels, telescope-temperature-dependent biases and a longitude-dependent bias, a mean instrument bias $\lesssim 0.6 \mathrm{~ms}^{-1}$ has been estimated for these data relative to northern-midlatitude radiosondes and to NWP models (Rennie and Isaksen, 2020; Martin et al., 2021).

Aeolus measures horizontal line-of-sight (HLOS) winds relative to the lidar boresight ${ }^{2}$. However, atmospheric dynamics is usually described in a resolved zonal/meridional framework, and thus we convert our observations into this frame. Appendix A describes this conversion and estimates errors arising from it, concluding that in our study region (i) zonal winds are generally robust except at the very highest latitudes, but (ii) meridional winds are only useful when heavily averaged, and even then

\footnotetext{
${ }^{1}$ A major SSW occurred a few months after launch in January 2019, but this was during an early phase of instrument operations for which data are not reliable.

${ }^{2} \mathrm{~A}$ small vertical wind $w$ component is present in the output HLOS data, causing an error $\sim 1.32 w$. As $w$ is usually small relative to $u$ and $v$ except in extreme cases such as strong gravity wave activity, we assume this effect to be negligible.
} 
https://doi.org/10.5194/wcd-2021-16

Preprint. Discussion started: 30 March 2021

(c) Author(s) 2021. CC BY 4.0 License.

(c) (i)

Weather and

Climate Dynamics

Discussions

have significantly suppressed magnitudes, typically by a factor of about ten. Except in Appendix A, we generally refer to our projected zonal and meridional wind estimates in this study without qualifying that they are derived in this way, but this distinction is important and should be kept in mind. In particular, in several places in this study we scale Aeolus estimates of $v$ by this empirically-determined factor of 10; while we highlight this scaling in every case and treat the results sceptically, the true difference between Aeolus and real $v$ is likely to vary both spatially and temporally and any such scaling is at best an approximation.

Aeolus' vertical scan pattern changes regularly. While the instrument is capable of measuring wind speeds at heights up to $30 \mathrm{~km}$, in practice the height-spacing of individual profiles varies to accommodate both specific science objectives and a broader numerical weather prediction role. We regularly grid our data for analysis to remove the effects of irregular vertical spacing, but we cannot compensate for the varying maximum measurement altitude; as such, the maximum height level of our study varies significantly, typically between heights of $17-25 \mathrm{~km}$.

\subsection{Microwave Limb Sounder}

We use v5.0 Level 2 data from the Microwave Limb Sounder on NASA's Aura satellite. Launched in 2004, Aura flies in a sun-synchronous polar orbit with equator-crossing times of 01.30 and 13.30. MLS uses a limb-sounding technique to measure atmospheric microwave emissions in five spectral bands (Schoeberl et al., 2006; Waters et al., 2006). Temperature and pressure are obtained from the 118 and $239 \mathrm{GHz}$ bands, retrieved over the range $261-0.001 \mathrm{hPa}(\sim 10-100 \mathrm{~km})$. Vertical resolution is variable, monotonically improving from $5 \mathrm{~km}-3.6 \mathrm{~km}$ over the height range $10-25 \mathrm{~km}$ before monotonically worsening again to $5 \mathrm{~km}$ at $40 \mathrm{~km}$ (see e.g. Figure 6a of Wright et al., 2016). This is finer than most nadir-sounding instruments, but significantly coarser than Aeolus. Along-track resolution is $\sim 170 \mathrm{~km}$, and estimated temperature precision is $\sim 0.6 \mathrm{~K}$ in the UTLS (Livesey et al., 2015).

We also derive geostrophic winds from MLS data. These winds are computed from the MLS v5.0 Level 2 GPH product, which is itself primarily derived from MLS temperatures (Livesey et al., 2006). Our MLS winds are calculated on a $5^{\circ} \times 20^{\circ}$ latitude/longitude grid independently for each MLS pressure level and day, using the relation $u=-(g / f) d Z / d y$, where $Z$ is GPH, $y$ is the meridional distance between measurement bins, $f$ is the Coriolis parameter and $g$ is the acceleration downwards due to gravity (assumed to be $9.81 \mathrm{~ms}^{-1}$ ). Values estimated on this grid are then bilinearly interpolated back to the instrument scan track, again for each pressure level and day independently, in order to provide a spatial weighting roughly equivalent to that for MLS temperature.

Due to this significant spatiotemporal averaging, these geostrophic winds are very coarse relative to Aeolus. The calculation also inherently relies on an assumption of atmospheric geostrophy. In our study region, we expect the difference between geostrophic and true wind to increase with height due to the driving effects of processes such as gravity wave breaking, and MLS wind estimates at high altitudes in particular should be treated with caution. We use only zonal-mean zonal MLS wind in this study, which we validate against Aeolus, reanalysis and operational analysis zonal-mean zonal winds in the troposphere and stratosphere in Section 2.4. 
https://doi.org/10.5194/wcd-2021-16

Preprint. Discussion started: 30 March 2021

(C) Author(s) 2021. CC BY 4.0 License.
Weather and

Climate Dynamics

Discussions
L.

MLS data are unavailable for a large fraction of the 24th of December 2020, and the remaining data exhibit a markedly different zonal mean and height dependence when compared to the surrounding days. We believe that this difference is due to the partial data coverage rather than true geophysical differences. Accordingly, to remove the effect of this anomalous day from our analyses, this day has been replaced in all analyses by the mean of the 23rd and 25th December 2020.

\subsection{ERA5 and ECMWF Operational Analyses}

We use ERA5 reanalysis output (Copernicus Climate Change Service, C3S; Hersbach et al., 2020) and European Centre for Medium-range Weather Forecasting (ECMWF) operational analysis (hereafter 'OpAl' for brevity). OpAl is only used in Section 2.4, where zonal-mean zonal wind is shown to be almost identical to ERA5 at the heights and times considered in the zonal mean, and in the rest of the study we use ERA5 only.

ERA5 is a fifth-generation reanalysis product produced by the ECMWF. OpAl is broadly similar in concept, but use a current version of the assimilative IFS weather model - for this study, IFS Cycle 47r1 - rather than a fixed version as with ERA5, which uses IFS Cycle 41r2, a 2016 version of the operational model. Furthermore, OpAl assimilates Aeolus data, while ERA5 does not. Therefore, ERA5 data are independent of Aeolus, while OpAl data are constrained by it. ERA5 is however constrained by other data sources, including in this altitude range by aircraft, radiosondes and satellite radiance and bending angle data, and therefore should reflect broadly the same geophysical state.

We use several forms of ERA5 data:

- In Figures 1 - 4, Figure 6, and Appendix A, we use temperature and zonal and meridional wind fields stored on 137 levels spaced non-uniformly from the surface to $0.01 \mathrm{hPa}$. These have been downsampled to a spatial resolution of $1.5^{\circ}$ and to a 3 hour time resolution to facilitate analysis of comparatively large data volumes.

- In Figures 5, 9 and 10, we use GPH stored on 37 pressure levels spaced non-uniformly from the surface to $1 \mathrm{hPa}(\sim 48 \mathrm{~km}$ altitude), with daily (specifically, midnight UTC) temporal resolution and at the full spatial resolution of $0.25^{\circ}$.

- In Figure 10 and 11, we use the $2 \mathrm{~m}$ altitude temperature field, also on a $0.25^{\circ}$ grid.

Like all reanalyses and models, these datasets inherently exhibit meaningful differences from the observed atmospheric state, both systematic and random, particularly at short lengthscales and high altitudes. Although systematic assessments of high-altitude wind accuracy at the global scale are challenging to produce due to a lack of suitable observations and GPH is not a measurable quantity, an assessment of temperature relative to limb sounder data suggest that, at the altitudes considered here, pointwise differences from observations were generally small, with typical pointwise root-mean-square temperatures differences from limb sounder observations $<2 \mathrm{~K}$ and Pearson linear correlations $>0.95$ (Wright and Hindley, 2018).

ERA5 data are only available for complete months ending at least two months before time of access. For periods closer to the date of access than this, an interim product known as ERA5T is made available at a five-day lag, which is produced in the same way as standard ERA5. This product is typically expected to exhibit no differences from the final product, but this is not guaranteed. Based on our date of article submission, we use ERA5T for all dates after the beginning of 2021, but due 

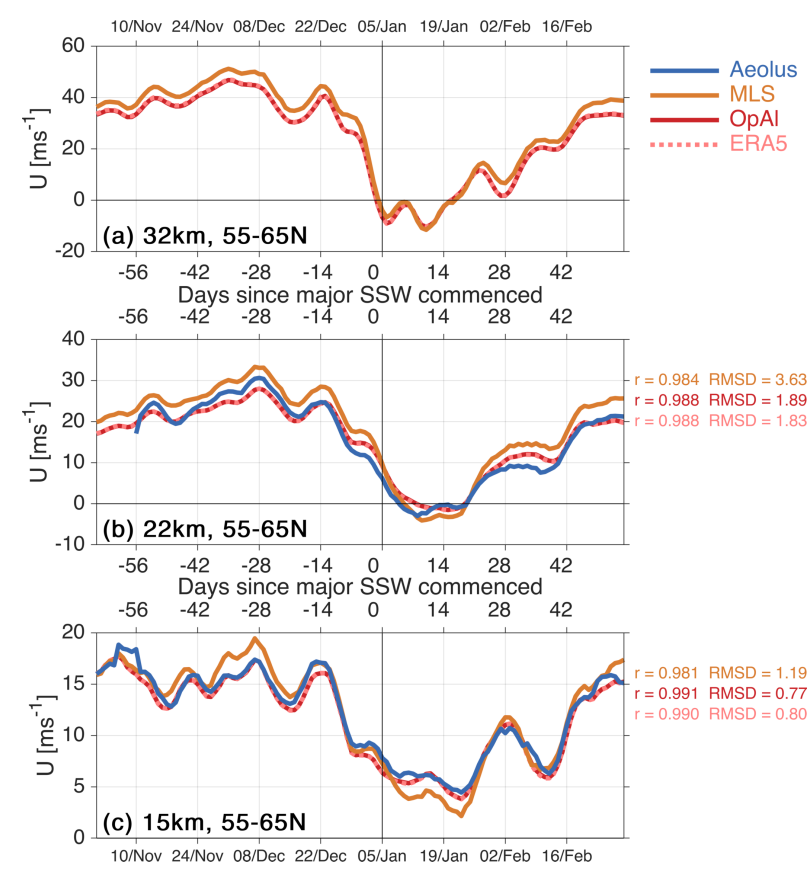

Figure 1. Daily-mean time series of zonal mean Aeolus HLOS-projected $u$, MLS geostrophic $u$, ERA5 $u$ and ECMWF operational analysis $u$ at (a) $32 \mathrm{~km}\left[\sim 10 \mathrm{hPa}\right.$ (b) $22 \mathrm{~km}$ and (c) $12 \mathrm{~km}$ altitude, averaged over the $55^{\circ}-65^{\circ} \mathrm{N}$ latitude band and smoothed three days to account for variable spatial coverage of satellite data. For each non-Aeolus datasets, the Pearson linear correlation with and root-mean-square difference (in $\mathrm{ms}^{-1}$ ) from the Aeolus time series is shown, computed over all days for which both datasets have coverage in that time series.

to the expected high level of similarity with the final ERA5 product refer to the two datasets interchangeably as ERA5. If this manuscript is accepted for WCD, we will replace these data with ERA5 during the revision process, highlighting any output changes at that time in our reviewer-response and removing this paragaph.

\subsection{Geostrophic Wind Validation}

Figure 1 shows time series of zonal mean zonal wind speed $\bar{u}$ derived from Aeolus (blue), ERA5 (pink dashed), OpAl (red), and MLS (orange), all averaged over the $55^{\circ}-65^{\circ} \mathrm{N}$ latitude band and shown at the (Figure 1a) $32 \mathrm{~km}$, (1b) $22 \mathrm{~km}$ and (1c) $15 \mathrm{~km}$ altitude levels. While this Figure shows clear evidence of the large dynamical changes due to the SSW that are the focus of our study, our primary purpose here is to cross-validate these four datasets, and we therefore reserve detailed discussion of the dynamical situation to Section 3 onwards. Aeolus data are not available in the top height level, and only commence in mid-November in the middle level.

Very close agreement is seen between the four time series, with Aeolus-relative correlations $\geq 0.98$ in all cases and rootmean-square-differences of $\leq 3.70 \mathrm{~ms}^{-1}$. Good visual agreement is also seen between MLS and reanalysis/operational analysis in the $32 \mathrm{~km}$ data. Based on this comparison, we conclude that MLS $\bar{u}$ is sufficiently robust in the zonal mean for use in our study, at least at these altitudes and latitudes; this reinforces the results of the manney previous studies that have used these 
https://doi.org/10.5194/wcd-2021-16

Preprint. Discussion started: 30 March 2021

(c) Author(s) 2021. CC BY 4.0 License.

(c) (i)

Weather and

Climate Dynamics

Discussions
L.

data to understand SSW dynamics (e.g. Manney et al., 2008, 2009, 2015). We further conclude that ERA5 and OpAl data in the zonal mean are sufficiently similar that they can be used interchangeably for our purposes; since some later analyses involve comparisons to climatology, we therefore use ERA5 throughout the remainder of this study for consistency.

Some differences are seen between the datasets, but these differences are small - this is consistent with the high quality of the datasets and very large spatial averaging implicit in taking a zonal mean. In particular, at the $22 \mathrm{~km}$ level both of the assimilative model datasets, i.e. ERA5 and OpAl, see $\bar{u}$ passing below $0 \mathrm{~ms}^{-1}$ a few days after the observational datasets. They also underestimate both local maxima and minima in $\bar{u}$ during the period after this, including missing a brief reversal seen by Aeolus but not MLS. Closer investigation of these discrepancies, including their geographic structure at spatial scales below the zonal mean, is beyond the scope of our study but may be a fruitful path for future work.

\section{The Climatological Context of the 2021 SSW}

While Aeolus launched in 2018 and was observing the atmosphere during winter 2018/19, due to laser instrument sensitivity issues and associated tests data products are only reliable from late June 2019 onwards. Furthermore, data for the 2019/20 winter remain unavailable due to reprocessing requirements. Therefore, we are only able to consider data from winter 2020/21 in this study. However, it is important to place this winter in context, in order to ascertain how representative our results are of winters in general and SSWs more specifically.

Figure 2 shows (a,c,e) MLS zonal mean temperature $\bar{T}$ and (b,d,f,g) ERA5 $\bar{u}$, analysed relative to a climatology produced using all winters between 2004/05 and 2019/20, i.e. the period since MLS began collecting data. $\bar{T}$ has been averaged across the entire region poleward of $60^{\circ} \mathrm{N}$ to provide an estimate of the mean polar context, while $\bar{u}$ has been averaged zonally across the $55^{\circ} \mathrm{N}-65^{\circ} \mathrm{N}$ latitude band to focus on the strength of the vortex-edge winds.

Figure 2 shows data at the (a,b) $20 \mathrm{~km}$, (c,d) $15 \mathrm{~km}$, (e,f) $10 \mathrm{~km}$ and (g) $5 \mathrm{~km}$ levels for all polar winters. 2020/21 is shown in red. Grey shading illustrates the spread of the 2004/05-2018/19 climatology, with shades of grey indicating specific positions in the climatology and with the 0th, 18th, 50th, 82nd and 100th percentiles (i.e. non-parametric equivalents of the range, median, and first and second standard deviations) emphasised by solid grey lines. For a fifteen-winter climatology such as this, the regions above and below the 18th and 82nd percentiles represent three individual winters each, while the region between these bounds represents the remaining nine winters. There have been two previous early-January major SSWs since 2004 (Butler et al., 2017), reaching zero $\bar{u}$ at $10 \mathrm{hPa}, 60^{\circ} \mathrm{N}$ on the 6th of January 2013 and 2nd of January 2019 respectively. We therefore broadly expect a 'typical' SSW to fall somewhere above the 82 nd percentile for temperature and below the 18th percentile for wind relative to this climatological period, but not to dramatically exceed the full range.

$\bar{T}$ before early December is below the central climatological range at the $20 \mathrm{~km}$ and $15 \mathrm{~km}$ level, rarely exceeding the $18 \mathrm{th}$ percentile. At the $10 \mathrm{~km}$ level, it lies in the central portion of the distribution, with a small excursion to a brief record minimum in mid-December. $\bar{u}$ during this period at altitudes $\geq 15 \mathrm{~km}$ is significantly above climatology to a similar degree as $\bar{T}$ is above; this is physically consistent, as it represents a strong vortex boundary which isolates the pole from midlatitudes and allows it 
https://doi.org/10.5194/wcd-2021-16

Preprint. Discussion started: 30 March 2021

(c) Author(s) 2021. CC BY 4.0 License.

(c) $\underset{\mathrm{Br}}{\mathrm{B}}$

Weather and

Climate Dynamics

Discussions
L.
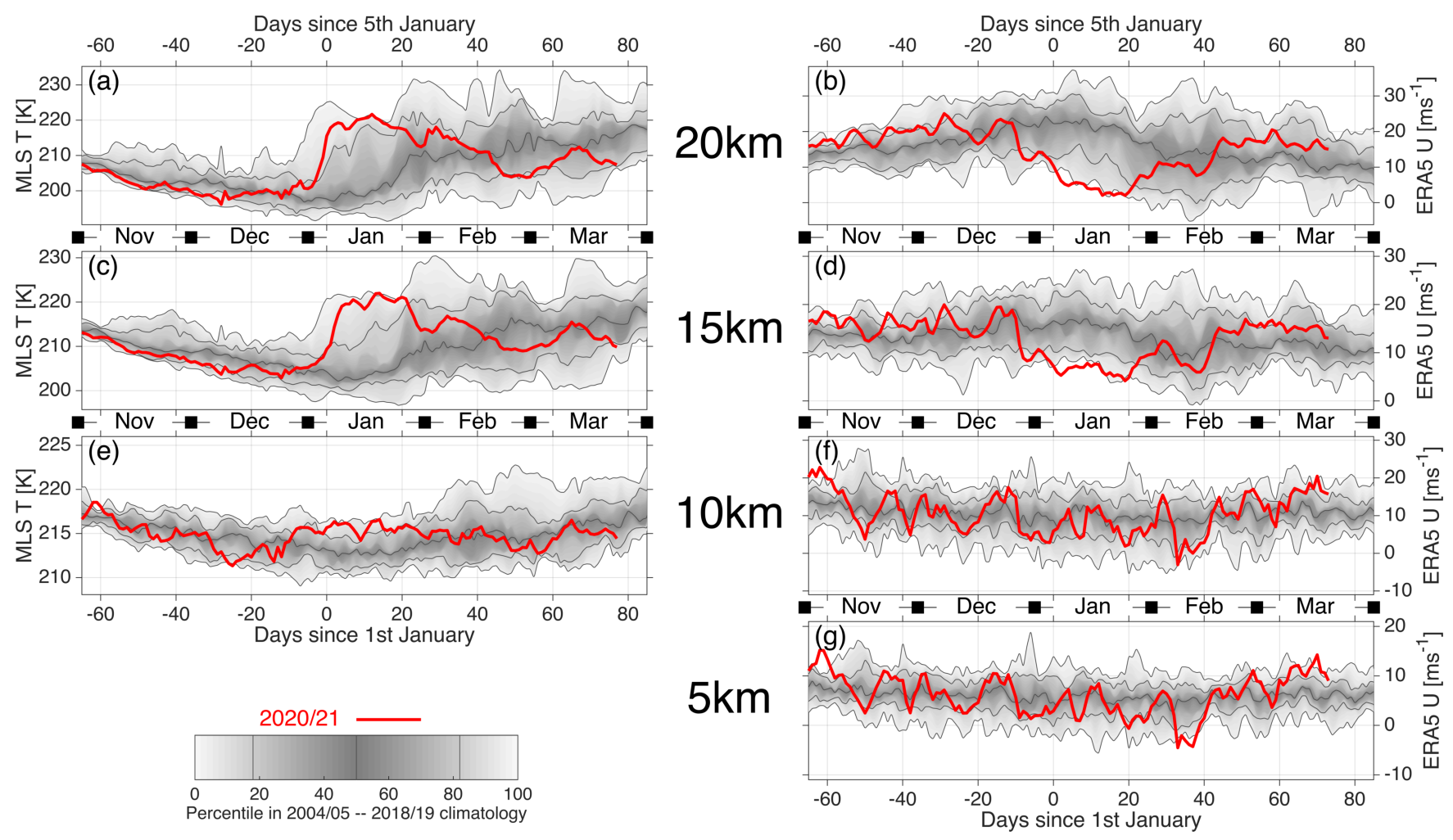

Figure 2. Context of the January 2021 SSW in relation to a 2004/05-2019/20 climatology of zonal mean (a,c,e) MLS temperatures and (b,d,f,g) ERA5 zonal winds at (a,b) $20 \mathrm{~km}(\mathrm{c}, \mathrm{d}) 15 \mathrm{~km}(\mathrm{e}, \mathrm{g}) 10 \mathrm{~km}$ and (g) $5 \mathrm{~km}$ altitude. Grey shading shows the climatological distribution. Red line shows winter 2020/21 within this context.

to radiatively cool, suggesting that the polar vortex was anomalously strong in this period. $\bar{u}$ below $15 \mathrm{~km}$ show no consistent trend, varying for example from an all-climatology maximum in early November to a near-minimum two weeks later.

From early December to around the 1 st of January at heights $\geq 15 \mathrm{~km}, \bar{T}$ starts to rise slightly, while $\bar{u}$ drops significantly. Around the 1 st of January, the rise in $\bar{T}$ rapidly accelerates, moving from the centre of the climatological distribution to nearrecord values by the 5th of January and remaining at this level for around 18 days. $\bar{u}$ during this period reaches a record minimum for this time period. After this maximum (minimum) in $\bar{T}(\bar{u})$, temperatures (winds) start to slowly return to normal; this return to normality is a mixture of falling temperatures during 2021 and a rise in the climatological median and range, and vice-versa for $\bar{u}$. After the first week of February, temperatures and winds at these altitudes remain within the central region for the remainder of the study period.

At altitudes $\leq 10 \mathrm{~km}$, temperatures rise above the 82 nd percentile following the SSW, fall back below this percentile around 20 days later and remain near the centre of the distribution for the rest of the period. $\bar{u}$ at these heights shows no major response to the first minimum of the SSW in early January. A minimum in $\bar{u}$ is seen around day 35 of the year at the $5 \mathrm{~km}$ level, which may be associated with a second $\bar{u}$ minimum associated with the SSW shown later in our study (Figures 4 and 8). 
https://doi.org/10.5194/wcd-2021-16

Preprint. Discussion started: 30 March 2021

(c) Author(s) 2021. CC BY 4.0 License.

\section{(c) (i)}

Weather and

Climate Dynamics

Discussions
L.

In general therefore, we conclude that the 2020/21 SSW was broadly typical for an SSW in the period since (at least) 2004 in terms of zonal-mean effects on UTLS polar dynamics, with winds and temperatures at or near date-record values but not dramatically exceeding them.

\section{Zonal-Mean Winds and Temperatures in the Winter 2020/21 Sudden Stratospheric Warming}

Figures 3 and 4 show MLS- and Aeolus-derived $\bar{u}$ and $\bar{T}$ for winter 2020/21, again averaged over the region poleward of $60^{\circ} \mathrm{N}$ for temperatures and over the $55^{\circ}-65^{\circ} \mathrm{N}$ latitude band for winds. Temperatures are shown as zonal mean anomalies $\overline{T_{a}}$ from the the mission-to-date day-of-year median, i,e, the difference between 2020/21 zonal-mean temperature and median climatological zonal-mean temperature; this is intended to both remove the strong vertical dependence of temperature on height and contextualise the data in the historical record.

For Aeolus $\bar{u}$ (Figure $4 \mathrm{~b}$ ), the bottom few kilometres represent an incomplete zonal mean (for example, Greenland reaches maximal altitudes of $>3 \mathrm{~km}$ in this latitude band) and a more technically challenging measurement than at higher altitudes. Thus, altitudes below $2 \mathrm{~km}$ have been omitted completely and altitudes below $\sim 5 \mathrm{~km}$ should be treated with caution, although we note that case studies using Aeolus data have shown plausible results at even the lowest altitudes (e.g. Banyard et al., 2021).

Times are shown as days relative to the 5th of January 2021. Aeolus data have been binned onto a $2 \mathrm{~km}$ by one-day grid. For MLS, daily time bins are also used, but the width of the height bins is set to $4 \mathrm{~km}$ at altitudes below $55 \mathrm{~km}$ altitude and $6 \mathrm{~km}$ above. The green and purple dot-dashed lines overlaid on the data shows the zonal-mean temperature-tropopause and -stratopause height at $60^{\circ} \mathrm{N}$ for that day, derived from ERA5 as described by Wright and Banyard (2020) and France et al. (2012) respectively. A wider latitude range $\left(50^{\circ} \mathrm{N}-70^{\circ} \mathrm{N}\right)$ was also tested for Aeolus winds; results were broadly similar but with a deeper and longer-lasting period of negative-zonal winds at the top of the measured column following the SSW.

\subsection{Stratospheric and Mesospheric Context}

We consider first the broader-scale picture provided by Figure 3. Polar-cap temperature (Figure 3a) is typical for the time of year in November, with $\left|\overline{T_{a}}\right|<5 \mathrm{~K}$ at all heights. $\overline{T_{a}}$ is small and negative in the mid-stratosphere and mid-mesosphere and small and positive around the stratopause and (in early November) in the upper mesosphere. The small local maximum around the stratopause is likely due to interannual stratopause height variability rather than a meaningful difference. Vortex-edge winds (Figure 3b) during this period are generally strong and zonal throughout the stratosphere and mesosphere, but with a noticeable dip in mid-November, particularly in the mid-mesosphere.

From around the 1st of December, mid-stratospheric $\overline{T_{a}}$ dips to more than $5 \mathrm{~K}$ below climatology for a few days, while mesospheric $\overline{T_{a}}$ becomes anomalously positive up to above $80 \mathrm{~km}$ altitude. These signals, while small, mark the start of a period of significant disruption at all heights, associated with a steady drop in stratopause height. Throughout early December, stratospheric temperature is significantly above climatology, reaching short-lived peaks of $\overline{T_{a}}>10 \mathrm{~K}$. In the mid-mesosphere, similar-magnitude negative anomalies are seen, with corresponding timing. $\bar{u}$ during this period alternately speeds up and slows down by a significant fraction, with the timing of these changes well-correlated with the temperature changes (and with 

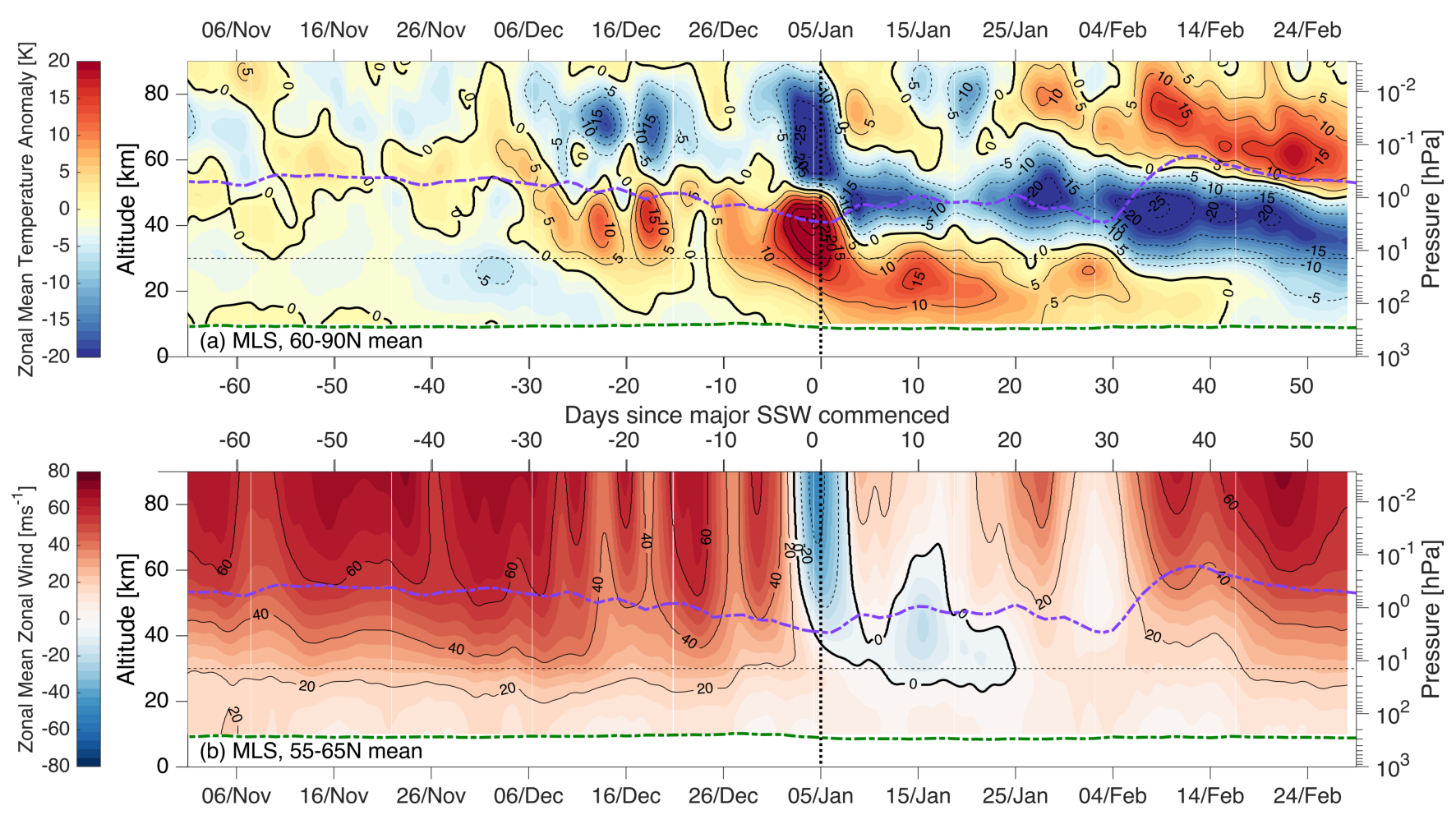

Figure 3. MLS-derived (a) $60^{\circ} \mathrm{N}-90^{\circ} \mathrm{N}$ mean temperature anomalies; (b) $55^{\circ} \mathrm{N}-65^{\circ} \mathrm{N}$ mean geostrophic zonal winds over the height range 0-90 km for winter 2020/21. Mean tropopause (stratopause) height derived from ERA5 is shown as a dot-dashed green (purple) line. Vertical dotted line indicates the date at which $10 \mathrm{hPa}$ winds reversed and the SSW became defined as major. Horizontal dashed line indicates the top of Figure 4.

changes in tropospheric eddy heat flux $v^{\prime} T^{\prime}$, shown and discussed in Section 6). This variability is strongly associated with the vortex beginning to distort and break up (Section 7); these modulations may be related to the polar-vortex vacillations which have been hypothesised to precede regime transitions from a strong to a weak vortex state (e.g. Scott, 2016).

From the 20th of December, $\overline{T_{a}}$ returns to normal for around five days at all heights, with $\left|\overline{T_{a}}\right|<5 \mathrm{~K}$ at all heights except for a small local maximum at the stratopause and with strengthened winds. This quiescent period is immediately followed by dramatic changes associated with the SSW which completely change the temperature and wind structure of the entire atmospheric column.

The developing SSW is seen in both the stratosphere and mesosphere, in both wind and temperature. $\overline{T_{a}}$ begins to rise in the stratosphere and fall in the mesosphere from the 26th of December, accelerating sharply from the 1st of January to reach a maximum (minimum) on the 4 th of January with anomalies $\left|\overline{T_{a}}\right|>25 \mathrm{~K}$ from climatology. Coincident with this, $\bar{u}$ at $60^{\circ} \mathrm{N}$ rapidly reverses over a few days at all heights above $\sim 30 \mathrm{~km}$, reaching zero at $10 \mathrm{hPa}(32 \mathrm{~km})$ on the 5 th of January. This region of negative $\bar{u}$ appears to extend through the stratosphere and mesosphere and continue above the top of the analysed region at $\sim 90 \mathrm{~km}$. Although MLS data are not validated for the top of our height range, a highly anomalous $u=-40 \mathrm{~ms}^{-1}$ signal can 
https://doi.org/10.5194/wcd-2021-16

Preprint. Discussion started: 30 March 2021

(c) Author(s) 2021. CC BY 4.0 License.

(c) (i)

Weather and

Climate Dynamics

Discussions

be seen during this period at altitudes of $80 \mathrm{~km}$ and above in data from the Esrange meteor radar $\left(68^{\circ} \mathrm{N} 21^{\circ} \mathrm{E}\right.$, Supplementary Figure S3), suggesting that the MLS signal is likely real.

After the zero-wind SSW criterion is reached, the stratosphere and mesosphere split into three distinct height-separated regimes, distinguished from each other by very different temporal patterns of $\overline{T_{a}}$ :

- In the lower stratosphere, $\overline{T_{a}}$ slowly returns to climatology, dropping to $<10 \mathrm{~K}$ by day $+20,<5 \mathrm{~K}$ by day +30 , and returning to climatology by late February (day +45$)$. This corresponds to an extended period of low $\bar{u}$ at these altitudes, with $|\bar{u}|$ only rarely exceeding $10 \mathrm{~ms}^{-1}$. The upper limit of this region slowly descends with time.

- Above $60 \mathrm{~km}$, temperature initially falls, reaching $\left|\overline{T_{a}}\right|<5 \mathrm{~K}$ by day +5 and $-10 \mathrm{~K}$ by day +15 . After this date we see a sharp rise, with $\overline{T_{a}}>10 \mathrm{~K}$ by day +22 and remaining consistently above this throughout February, again descending slowly with time. Variations in $\bar{u}$ roughly correlate with $\overline{T_{a}}$ in this region.

- Around the stratopause, $\overline{T_{a}}$ initially falls rapidly, dropping $>45 \mathrm{~K}$ in three days in a mirror to the sharp pre-SSW rise. This decline slows at around day +4 and reverses at day +13 , after which $\overline{T_{a}}$ begins to fall to reach $<-15 \mathrm{~K}$ by day +22 . This drop coincides with rising $\overline{T_{a}}$ in the mesosphere. From day +22 onwards, the temporal evolution of $\overline{T_{a}}$ closely mirrors the mesosphere for the rest of the studied period, with a descending boundary between the two regions related to a change in stratopause height discussed below. $\bar{u}$ in this region is only weakly correlated with $\overline{T_{a}}$.

Around day +30 , the mean stratopause height rapidly jumps upwards by around $20 \mathrm{~km}$; this is consistent with previous studies of upper-stratospheric SSW effects (e.g. Siskind et al., 2007; Manney et al., 2008; Wright, 2010; Wright et al., 2010; Manney et al., 2015), and is due to a new stratopause forming at high latitudes and altitudes rather than a sudden jump in the height of the original equator-connected stratopause (shown in zonal-mean MLS temperature in Supplementary Figure S1), likely due to the filtering out of orographic gravity waves by the lower-vortex near-zero winds. After this transition, the new stratopause continues to slowly descend for the rest of the studied period, forming a boundary between an unusually-cold stratosphere and an unusually-warm mesosphere. Tropopause height also exhibits a small amount of variability, falling by around $2 \mathrm{~km}$ immediately after the SSW begins and remaining below the pre-SSW height for the rest of the study period.

\subsection{Winds and Temperatures in the UTLS and Troposphere}

We next consider tropospheric and lower stratospheric $\bar{u}$ as measured by Aeolus, Figure $4 \mathrm{~b}$. MLS $\overline{T_{a}}$ is shown over the same height range in Figure 4a to help place our Aeolus results within the context of Figure 3. Note however that the vertical resolution of MLS in this height range is poor, with only 6 independent height bins in the range shown here. Appendix B shows and discusses the same fields derived from ERA5 data, for comparison.

Strong positive $\bar{u}$ is seen in the lower stratosphere from the beginning of November until the last week of December, with slower but still positive $\bar{u}$ in the troposphere except in the lowest few kilometres of our data. Variations in $\bar{u}$ occur approximately uniformly across the observed height range. 
https://doi.org/10.5194/wcd-2021-16

Preprint. Discussion started: 30 March 2021

(c) Author(s) 2021. CC BY 4.0 License.

Weather and

Climate Dynamics

Discussions

L.
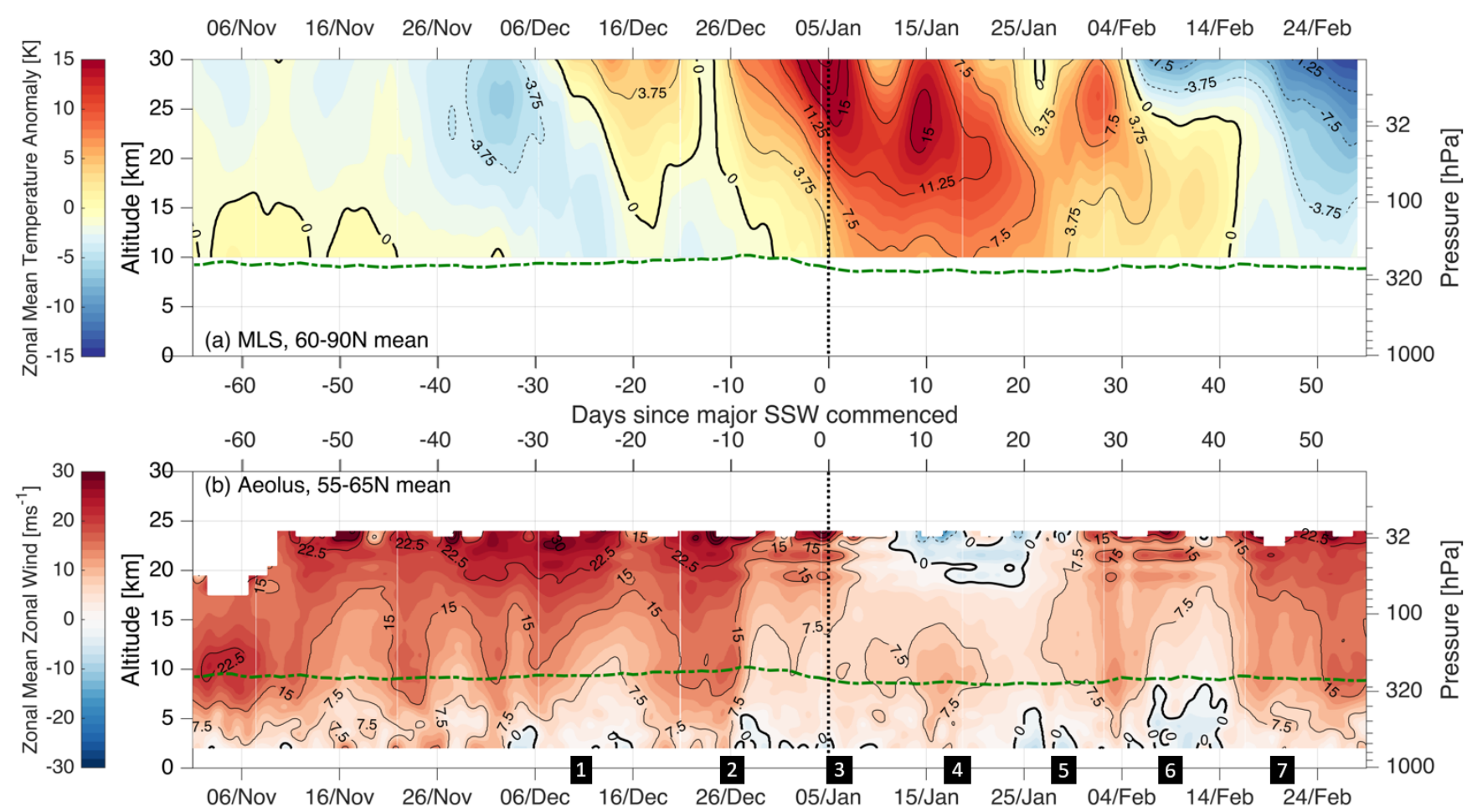

Figure 4. (a) MLS-derived $60^{\circ} \mathrm{N}-90^{\circ} \mathrm{N}$ mean temperature anomalies; (b) Aeolus-derived $55^{\circ} \mathrm{N}-65^{\circ} \mathrm{N}$ mean projected zonal winds for winter 2020/21, for altitudes 0-30 km. Mean tropopause height derived from ERA5 is shown as a dot-dashed green line. Vertical dotted line indicates the date at which $10 \mathrm{hPa}$ winds reversed and the SSW became defined as major. Numbered boxes on the horizontal axis of (b) are used to guide discussion from Section 7.1 onwards.

Starting around the 6th of December, $\bar{u}$ falls at all heights for around a week. This is approximately coincident with a similar short burst of increased $\overline{T_{a}}$, suggesting that weakened winds are allowing some warming of the vortex; this $\bar{u}$ and subsequent $\overline{T_{a}}$ feature propagates upwards from lower-tropospheric altitudes (Figure $4 \mathrm{~b}$ ) rather than downwards from the middle atmosphere (Figure 3b). Winds begin to increase in speed again around the 16th of December, this time with increased $\bar{u}$ propagating downwards from above, and stratospheric $\overline{T_{a}}$ similarly returns to normal.

From the 26th of December, a sharp drop in $\bar{u}$, from a typical speed in the UTLS of $15 \mathrm{~ms}^{-1}$ to around $5 \mathrm{~ms}^{-1}$, is seen at all heights below $20 \mathrm{~km}$. This marks the beginning of the SSW proper, as stratospheric $\overline{T_{a}}$ also starts to rapidly increase at this date. As the SSW becomes major and the positive $\overline{T_{a}}$ anomaly begins to propagate downwards into the lower stratosphere, we also see rapidly-dropping $\bar{u}$ at the top of the Aeolus column, corresponding temporally with the same drop in MLS wind seen in Figure 3b. $\bar{u}$ at $25 \mathrm{~km}$ altitude, the highest level observed by Aeolus, reverses (i.e. drops below zero) five days after it does so at $10 \mathrm{hPa}$, with the zero wind line descending to reach a minimum altitude on the 15 th of January, i.e. day +10 from the SSW. 


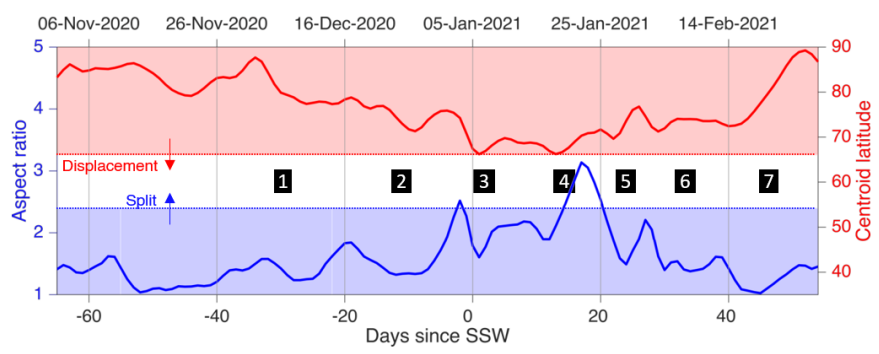

Figure 5. (red line) Centroid latitude (blue line) aspect ratio of the polar vortex around the time of the January 2021 SSW, computed from ERA5 GPH at the $10 \mathrm{hPa}$ level following the method of Seviour et al. (2013). Unshaded region indicates anomalous values indicative of a major vortex distortion, i.e. a split vortex for the blue line or a displaced vortex for the red line. Numbered boxes across the centre of the plot are used to guide discussion from Section 7.1 onwards.

From day +10 to day +20 , the descending zero $\bar{u}$ contour stalls at a minimum altitude of $19 \mathrm{~km}$. $\bar{u}$ below this remains suppressed, with the local maximum around the tropopause returning to pre-SSW levels around day +17 . During this period, $\overline{T_{a}}$ is large throughout the lower stratosphere, remaining above $+7.5 \mathrm{~K}$ at most heights. This period of reduced winds corresponds to a period where the vortex is displaced by over $20^{\circ}$ from the pole for an extended period, discussed in Sections 8 and 7.2 below.

On days $+21-+22$, wind speeds throughout the column begin to increase again, rising by around $15 \mathrm{~ms}^{-1}$ at day +30 at all heights above $5 \mathrm{~km}$ altitude. This value represents a local maximum, after which $\bar{u}$ again reduces to reach a minimum at all heights on day +40 . This reduction is likely a downward-propagating response to a second drop below zero $\bar{u}$ at $10 \mathrm{hPa}$ seen in reanalysis and operational analysis winds on the 4th of February (day +30; Figure 1a); note that, while a local minimum is also seen at this date in MLS $\bar{u}$ (Figures 1a, 3b), observations do not quite reach zero. This minimum is near-zero in the lower stratosphere and below zero in the troposphere, but larger at the highest altitudes measured by Aeolus, and thus may represent a mixture of processes.

Finally, from day +40 onwards, wind speeds throughout the Aeolus column start to increase, and remain high for the rest of the study period. This represents a steady transition back to a typical winter vortex, as seen in Section 3 using in reanalysis data.

\section{Split or Displacement?}

SSWs are often categorised into splits, where the polar vortex divides into two or more sub-vortices, and displacements, where the vortex shifts off the pole but does not split (Charlton and Polvani, 2007; Mitchell et al., 2013). This distinction is important, as there is growing evidence from both model and observational studies that both the triggering processes and weather impacts of these two types of event can be quite dissimilar. 
https://doi.org/10.5194/wcd-2021-16

Preprint. Discussion started: 30 March 2021

(c) Author(s) 2021. CC BY 4.0 License.

(c) (i)

Weather and

Climate Dynamics

Discussions

Figure 5 investigates the split/displacement nature of the early 2021 SSW, based on applying the vortex-moment diagnostics of Seviour et al. (2013) to daily-averaged ERA5 GPH at the $10 \mathrm{hPa}$ level. The estimated centroid latitude of the polar vortex is shown as a red solid line and the aspect ratio of the vortex as a blue solid line. By applying threshold criteria to these values, this method allows us to classify SSW events as a split or a displacement.

Seviour et al. (2013) empirically defined splits as events where the aspect ratio (blue line) remained $\geq 2.4$ for seven days or more, and displacement events as those where the centroid latitude (red line) dropped equatorward of $66^{\circ}$ for more than seven days. Such classifications are typically made at the $10 \mathrm{hPa}$ level and we thus restrict our discussion here according; note that Figure 9, discussed below, shows some evidence of vortex-splitting taking place at the $70 \mathrm{hPa}$ level.

The January 2021 SSW meets neither the split-event nor the displacement-event criteria at the $10 \mathrm{hPa}$ level, but closely approaches both, making it a mixture of the two event types.

The vortex centroid latitude never moves equatorward of $66^{\circ}$, but is equatorward of $69^{\circ}$ for nine consecutive days from the 12th of January to the 20th of January. This period falls during the period of most negative zonal winds in the UTLS in our Aeolus data, and corresponds temporally to both strongly negative zonal winds at $60^{\circ} \mathrm{N}$ in MLS $\bar{u}$ at $32 \mathrm{~km}(\sim 10 \mathrm{hPa}$, Figure 3b) and in Aeolus $\bar{u} 10 \mathrm{~km}$ below this (Figure 4b).

The vortex aspect ratio exceeds 2.4 twice, on the 3rd of January for one day and starting on the 20th of January for six days, but never meets the seven-consecutive-day element of the Seviour et al. (2013) criteria. Given we are using daily-averaged data for this analysis, it is conceivable that there is a seven-day period offset from the diurnal cycle where the aspect ratio exceeds 2.4 which would allow us to tentatively class this SSW as a split warming; however, such a classification is marginal at best. Under the alternative criteria of Gerber et al. (2021), who first define events by zonal wind reversal and then subdivide into splits and displacements by the number of days in which the thresholds are exceeded within $+/-10$ days of onset, this event would be classified more directly as a split event.

\section{Mesoscale Wave Forcing, in Reanalysis and Observations}

The eddy heat flux $\overline{v^{\prime} T^{\prime}}$ and eddy momentum flux $\overline{u^{\prime} v^{\prime}}$, where the ' indicates deviations from the zonal mean and the overline an area-average, are widely used in atmospheric science to study the large-scale transport of heat and momentum by mesoscale waves and eddies. These parameters have significant implications for the stability of the polar vortex, and often prove useful in terms of forecasting how and when SSWs form. In particular, previous work has shown a clear relationship in reanalysis output whereby anomalously strong lower-stratospheric $\overline{v^{\prime} T^{\prime}}$ nearly always precedes weak vortex events such as SSWs (Polvani and Waugh, 2004).

As Aeolus data provide systematic global-scale measurements of tropospheric and lower stratospheric winds, they have the potential to help us estimate these fluxes entirely from observational data. To both assess this and to study the dynamical processes involved in this specific SSW, we here estimate $\overline{v^{\prime} T^{\prime}}$ and $\overline{u^{\prime} v^{\prime}}$ using Aeolus and MLS data and ERA5 output (Figure 6). These estimates are computed by first gridding the data onto a $2 \mathrm{~km} \times 30^{\circ} \times 10^{\circ}$ (height $\times$ longitude $\times$ latitude) grid, taking the difference of each point from the zonal mean, and then averaging the results over the $45^{\circ} \mathrm{N}-75^{\circ} \mathrm{N}$ region at each 
https://doi.org/10.5194/wcd-2021-16

Preprint. Discussion started: 30 March 2021

(c) Author(s) 2021. CC BY 4.0 License.

(c) (1)

Weather and

Climate Dynamics

Discussions

L
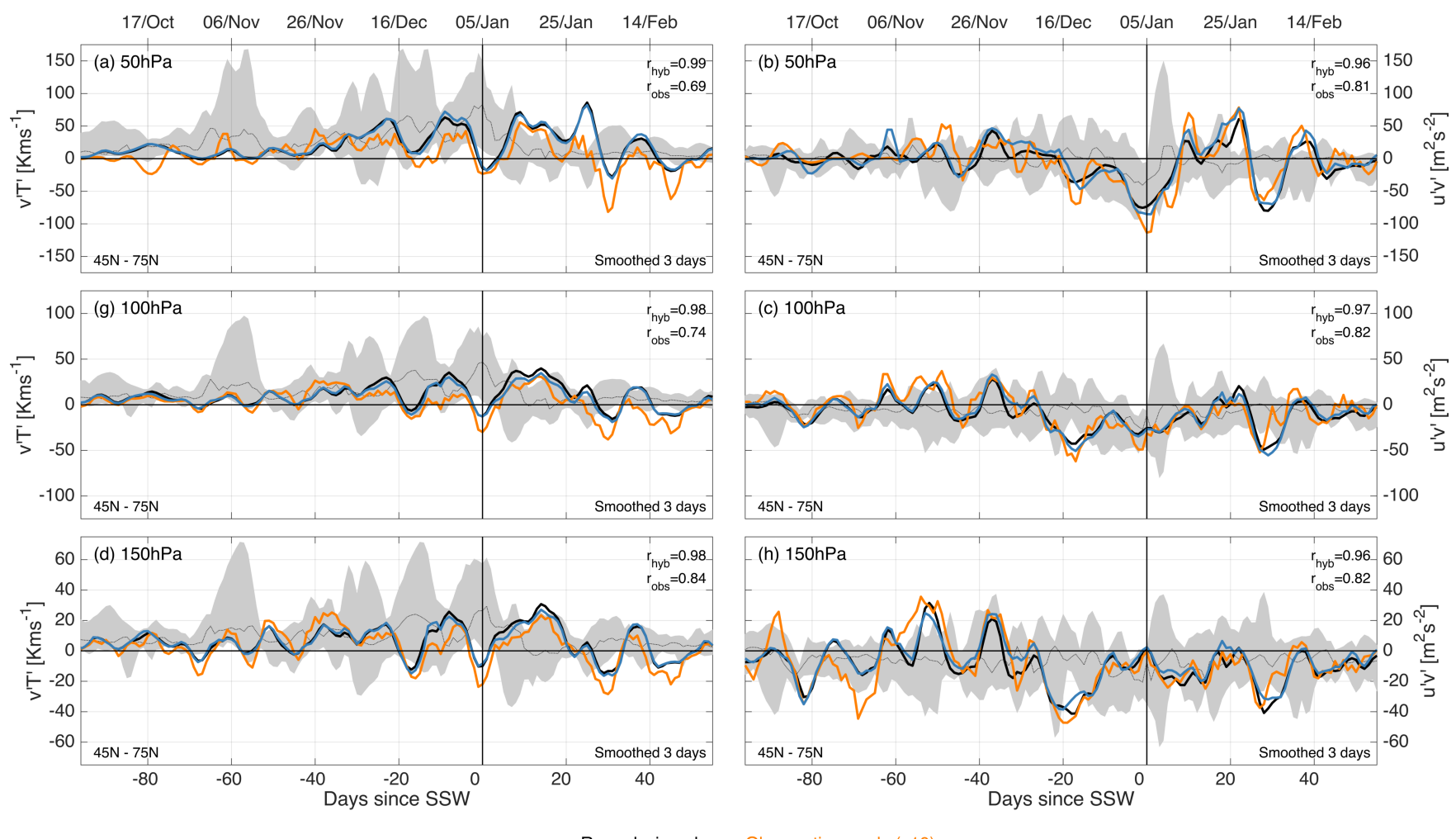

Reanalysis-only

Hybrid

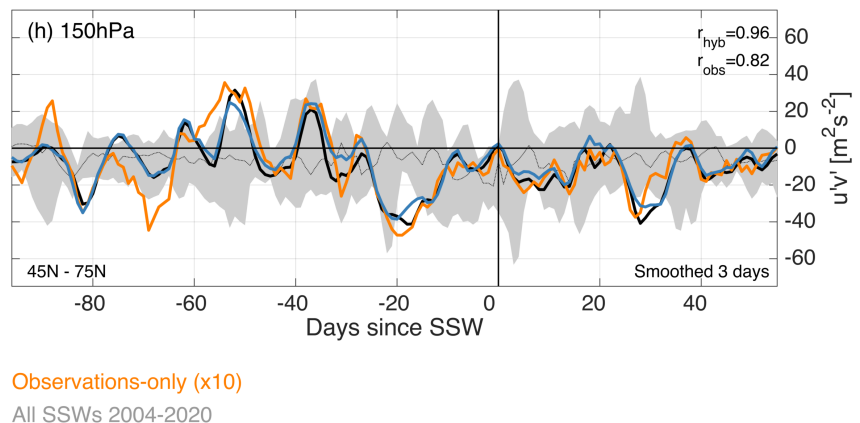

Figure 6. Eddy heat fluxes $\overline{v^{\prime} T^{\prime}}$ and eddy momentum fluxes $\overline{u^{\prime} v^{\prime}}$ estimated from MLS temperature data, Aeolus wind data and ERA5 wind and temperature output, for three different combinations of these data sources: 'ReA' (all-reanalysis, black), 'Obs' (all-observed, orange) and 'Hyb' ("hybrid", observed $u$ and $T$ with reanalysis $v$, blue). Data shown are averaged over the $45^{\circ} \mathrm{N}-75^{\circ} \mathrm{N}$ latitude range, and shown at the (a,b) $50 \mathrm{hPa},(\mathrm{c}, \mathrm{d}) 100 \mathrm{hPa}$ and (e,f) $150 \mathrm{hPa}$ pressure levels. Observational series have been scaled by an empirically-chosen factor of $\times 10$. Pearson linear correlations $r$ of Obs and Hyb with ReA are indicated at the top right of each panel. Grey shading indicates the full range of reanalysis-only daily estimates from all major SSWs between the 2004 and 2020 boreal summers, with the median indicated by a black dotted line.

height to produce the time series shown. ERA5 output have been treated as individual points assigned to the centre of each model gridbox, in order to ensure full consistency in data treatment.

We compute three versions of $\overline{v^{\prime} T^{\prime}}$ and $\overline{u^{\prime} v^{\prime}}$. The first of these, labelled "ReA", is computed entirely from reanalysis data. Given the large spatial scales involved in the analysis we assume for the purposes of this discussion that ReA represents a value close to truth, and use it as a baseline for our other versions. Separately, we compute a fully-observational estimate, "Obs", using MLS and Aeolus $u, v$ and T. Finally, in light of the significant uncertainties in $v^{\prime}$ identified in Appendix A, we show a further estimate of $\overline{v^{\prime} T^{\prime}}$ and $\overline{u^{\prime} v^{\prime}}$, "Hyb", which is a hybrid estimate computed using Aeolus $u$, MLS T, and ERA5 $v$ 
https://doi.org/10.5194/wcd-2021-16

Preprint. Discussion started: 30 March 2021

(c) Author(s) 2021. CC BY 4.0 License.

Weather and

Climate Dynamics

Discussions

L.

355 to demon
earlier de
Figure
methodol
the reana
at the tim
data; corr
numerica
but which
To con
ERA-Inte
01-21, 2007
have each
grey shadin we
At the
of negati
several day
surroundi
represent earlier decision to only consider MLS geostrophic winds in the zonal mean (Section 2.2).

Figure 6a-f show (a,c,e) $\overline{v^{\prime} T^{\prime}}(\mathrm{b}, \mathrm{d}, \mathrm{f}) \overline{u^{\prime} v^{\prime}}$ time series at (a-b) $50 \mathrm{hPa}(\sim 21 \mathrm{~km}$ altitude) (c-d) $100 \mathrm{hPa}(\sim 16 \mathrm{~km})$ and (ef) $150 \mathrm{hPa}(\sim 14 \mathrm{~km})$. Observational series have been scaled by an empirically-chosen factor of 10 to compensate for the methodological low bias in Aeolus $v$ (Section 2). Pearson linear correlations between the hybrid and observed time series and alysis-only time series are shown at the top right of Figures 6a-f. All data have been boxcar-smoothed by three days time series level (i.e. after computation of all variables) and the correlations have been computed from the smoothed data; correlations of the unsmoothed daily data give proportionally similar results for the different time series but with smaller numerical values, particularly for Obs which exhibits fractionally large day-to-day variability due to sampling pattern issues Which is stable at the three-day-smoothed level.

contextualise these data, we also show estimated $\overline{u^{\prime} v^{\prime}}$ and $\overline{v^{\prime} T^{\prime}}$ for the nine SSWs since August 2004. Following the ERA-Interim derived estimates of Butler et al. (2017), we define these as having commencement dates of, consecutively, 200601-21, 2007-02-24, 2008-02-22, 2009-01-24, 2010-02-09, 2010-03-24, 2013-01-06, 2018-02-12 and 2019-01-02. These events have each been individually processed using the same approach as our 2021 ReA estimates and their full range is indicated by rey shading, with the black dotted line showing the distribution median.

At the start of October, $\overline{v^{\prime} T^{\prime}}$ is at the low end of the nine-SSW climatology at all levels. At $50 \mathrm{hPa}$, there is a brief burst of negative $\overline{v^{\prime} T^{\prime}}$ in Obs 80 day before the SSW, but not in ReA or Hyb. This deviation grows and shrinks smoothly over several days, lasts long enough that it is unlikely to be due to sampling bias, and represents a sign change in $v$ relative to the surrounding period which reprojecting HLOS wind into the $v$-direction should be robust against (Appendix A). Thus, this may represent a real difference between ERA5 and true $v$. A brief period of negative $\overline{v^{\prime} T^{\prime}}$ is also seen at the $100 \mathrm{hPa}$ and $150 \mathrm{hPa}$ ound day -50 , which is consistent across the three times series but with lower amplitude in Hyb and ReA. $\overline{u^{\prime} v^{\prime}}$ in r shows large oscillations about zero, but the amplitude of these oscillations is broadly consistent with our nine-SSW atology at all heights, and of roughly the same magnitude between levels (note the different vertical scales).

we move into November, we begin to see $\overline{v^{\prime} T^{\prime}}$ oscillations with periods of approximately one week in all three time t $150 \mathrm{hPa}$ level, overlying a positive overall trend. The $100 \mathrm{hPa}$ level shows similar behaviour with approximately the elative to the oscillation amplitude $\overline{u^{\prime} v^{\prime}}$ during November continues to oscillate significantly and with a larger amplitude than in October, with an underlying shift to a higher and then lower medium-term ( $\sim$ fortnightly) mean at all three levels.

Things begin to change in early December. In $\overline{v^{\prime} T^{\prime}}$, we see a positive overall trend from around day -40 until immediately before the beginning of the SSW in January. This trend, albeit interrupted by a sharp dip of around one week in mid-December, is visible and clear at all three levels and is consistent with our expectation of strong $\overline{v^{\prime} T^{\prime}}$ before an SSW. With the exception of the dip, values are almost exactly at the median for our nine-SSW climatology (black dotted line), with the same positive trend. While the dip represents an outlier from this trend, it is only slightly outside the full range of the climatology in the negative direction for a few days in Obs and not at all in ReA and Hyb. 
https://doi.org/10.5194/wcd-2021-16

Preprint. Discussion started: 30 March 2021

(c) Author(s) 2021. CC BY 4.0 License.

(c) (i)

Weather and

Climate Dynamics

Discussions
L

390

395

$\overline{u^{\prime} v^{\prime}}$ during this period also trends lower, and is also largely within climatology except for a brief period at lower levels which coincides with the dip in $\overline{v^{\prime} T^{\prime}}$; this suggests that the dip is being driven by changes in $v^{\prime}$ rather than $u^{\prime}$ or $T^{\prime}$. This dip in both series coincides with a brief shift in the $10 \mathrm{hPa}$ vortex towards a more elliptical vortex (Figure 5 ) centred at $80^{\circ} \mathrm{N}$ between north Greenland and Russia (Figure 9, discussed below), and the negative $v^{\prime}$ anomaly is consistent with disrupted flows during this period seen over Eurasia and the Pacific in maps of Aeolus wind at $17 \mathrm{~km}$ altitude (Figure 7, also discussed below).

At the beginning of January, all-height $\overline{v^{\prime} T^{\prime}}$ and $50 \mathrm{hPa} \overline{u^{\prime} v^{\prime}}$ both drop significantly, reaching their smallest values across the entire period to date. This drop in $\overline{u^{\prime} v^{\prime}}$ is at the lower limit of climatology for $\overline{u^{\prime} v^{\prime}}$ and significantly outside it for $\overline{v^{\prime} T^{\prime}}$, representing unusual mesoscale behaviour for the start of an SSW - in particular, the median of our climatology suggests that $\overline{v^{\prime} T^{\prime}}$ at this point would normally be at a maximum relative to several months on either side of the beginning of an SSW. This is anomalous, and may perhaps suggest that the initial increase in $\bar{T}$ could have been driven by the mean flow rather than eddies. After $\sim 10$ days the eddies do seem to drive a strong warming though.

A few days after the SSW formally commences, we see sharp rises in $\overline{v^{\prime} T^{\prime}}$ and $50 \mathrm{hPa} \overline{u^{\prime} v^{\prime}}$, back to slightly above pre-SSW values, moving from significantly below the climatological range to slightly above it, and from around the 10th of January $($ day +5$)$ until early February these values remain anomalously high, reaching a time-series maximum a few days after the vortex returns to its normal aspect ratio range at $10 \mathrm{hPa}$ following a six-day split (Figure 5). From this point, both variables begin oscillating strongly, and over the next two weeks they drop from a time-series maximum well above climatology to a time-series minimum well-below, then back to a maximum above. This degree of variability is highly anomalous, with the oscillations in this single year larger than the entire data range over this period seen around the last nine SSWs. The oscillations roughly follow shifts in the vortex aspect ratio and centroid location at the $10 \mathrm{hPa}$ level above (Figure 5), and correspond to varying zonal-mean upper-tropospheric winds (Figure 4) related to a short attempt by the vortex to return to its normal morphology which ultimately fails (Section 7.2.3). $\overline{u^{\prime} v^{\prime}}$ at the $100 \mathrm{hPa}$ and $150 \mathrm{hPa}$ levels differ from the other four time series for the January period, remaining well inside climatology, but share the precipitous drop and subsequent rise seen in early February.

Finally, in late February we see a return to typical post-SSW values. This is consistent with the largely normal wind speed seen during this period in e.g. Figures 3 and 4. As in October, $\overline{v^{\prime} T^{\prime}}$ is once again at the lower end of climatology but still within it, amd $\overline{u^{\prime} v^{\prime}}$ is close to the distribution median.

Based on these results, we draw three conclusions for future calculations of $\overline{v^{\prime} T^{\prime}}$ and $\overline{u^{\prime} v^{\prime}}$ using Aeolus-type wind data and equivalent supporting temperature data such as that from MLS, and two conclusions about mesoscale-eddy forcing during the 2021 SSW.

1. [Aeolus] Current one-dimensional observations with Aeolus and MLS can clearly identify the large relative changes in $\overline{v^{\prime} T^{\prime}}$ and $\overline{u^{\prime} v^{\prime}}$ associated with SSWs, albeit with a very large $(\sim \times 10)$ but consistent error in magnitude. Relative to reanalysis, the observations diverge from background at the same time, show the same oscillations both large and small, and correlate well (at three-day smoothing, $>0.8$ in most cases and $\sim 0.7$ at worst) given the potentially large pointwise errors in our inferred $u$ and $v$. 
2. [Aeolus] Hybrid products using reanalysis-derived $v$ show exceptional correlation with pure-reanalysis estimates, highlighting the high quality of Aeolus $u$ and MLS T.

3. [Aeolus] It is necessary to significantly spatially average Aeolus $v$ data to perform such an analysis. Calculations of $\overline{v^{\prime} T^{\prime}}$ and $\overline{u^{\prime} v^{\prime}}$ on narrower-spaced grids in latitude (not shown) exhibited much larger deviations from the reanalysis and hybrid estimates, consistent with the large uncertainty on $v$.

4. [Eddy-forcing] Eddy-forcing was normal for momentum flux but appears to be anomalus for heat flux, where we appear to see a time-minimum during the SSW onset. While we do not calculate a detailed heat budget here, if correct this may suggest that the increase in stratospheric temperatures near SSW onset are a result of changes in the mean flow rather than eddy-driven.

5. [Eddy-forcing] The January $2021 \mathrm{SSW}$ was also unusual in the degree of variability seen after the initial outbreak of negative $10 \mathrm{hPa}$ winds, with very large oscillations seen relative to climatology in $\overline{v^{\prime} T^{\prime}}$ and $\overline{u^{\prime} v^{\prime}}$. These oscillations approximately correspond temporally to an initial recovery of the vortex and subsequent return to SSW conditions for a few days.

\section{Vortex Structure at Sub-Zonal Scales}

While the zonal mean is often a useful tool for broadly characterising the atmospheric state, it can disguise important local variations in atmospheric structure. To better understand the dynamical changes to UTLS and tropospheric wind patterns induced by the 2020/21 SSW, in this Section we examine geographic variations in (a) Aeolus winds in the UTLS and troposphere and (b) ERA5 GPH at the $10 \mathrm{hPa}$ and $70 \mathrm{hPa}$ levels.

\subsection{The 3D Polar Vortex in Aeolus Winds}

Figures 7 and 8 illustrate the geographic structure of Aeolus-derived polar winds during the 2020/21 SSW in 2D and 3D respectively. For clarity of discussion we define seven approximate 'phases'; these phases are typified by the selected dates in Figure 8, each of which corresponds to multiple panels in Figure 7 identified by a labelled box for each phase. These phases (for time series, indicated at an approximate midpoint rather than either their commencement or the typical days shown in Figure 8) are also shown on Figures 4b, 5, 9 and 10 to provide context throughout the study.

Figure 7 shows Aeolus-derived $u$ and $v$ for consecutive five-day periods between December 2020 and February 2021 at $17 \mathrm{~km}$ altitude $^{3}$. This altitude is the highest where coverage consistently extends equatorward of $60^{\circ}$, allowing us to contextualise the vortex changes in the horizontal domain. Colours show zonal wind speed; vector arrows show relative wind speeds and absolute directions after $v$ has been scaled by a factor of 10 . Three-day-means were separately assessed, and gave broadly the

\footnotetext{
${ }^{3}$ At time of submission, a daily-animated version of this figure is available from the European Space Agency website, https://www.esa.int/Applications/ Observing_the_Earth/Aeolus/Aeolus_shines_a_light_on_polar_vortex
} 
https://doi.org/10.5194/wcd-2021-16

Preprint. Discussion started: 30 March 2021

(C) Author(s) 2021. CC BY 4.0 License.

Weather and

Climate Dynamics

(c) (i)
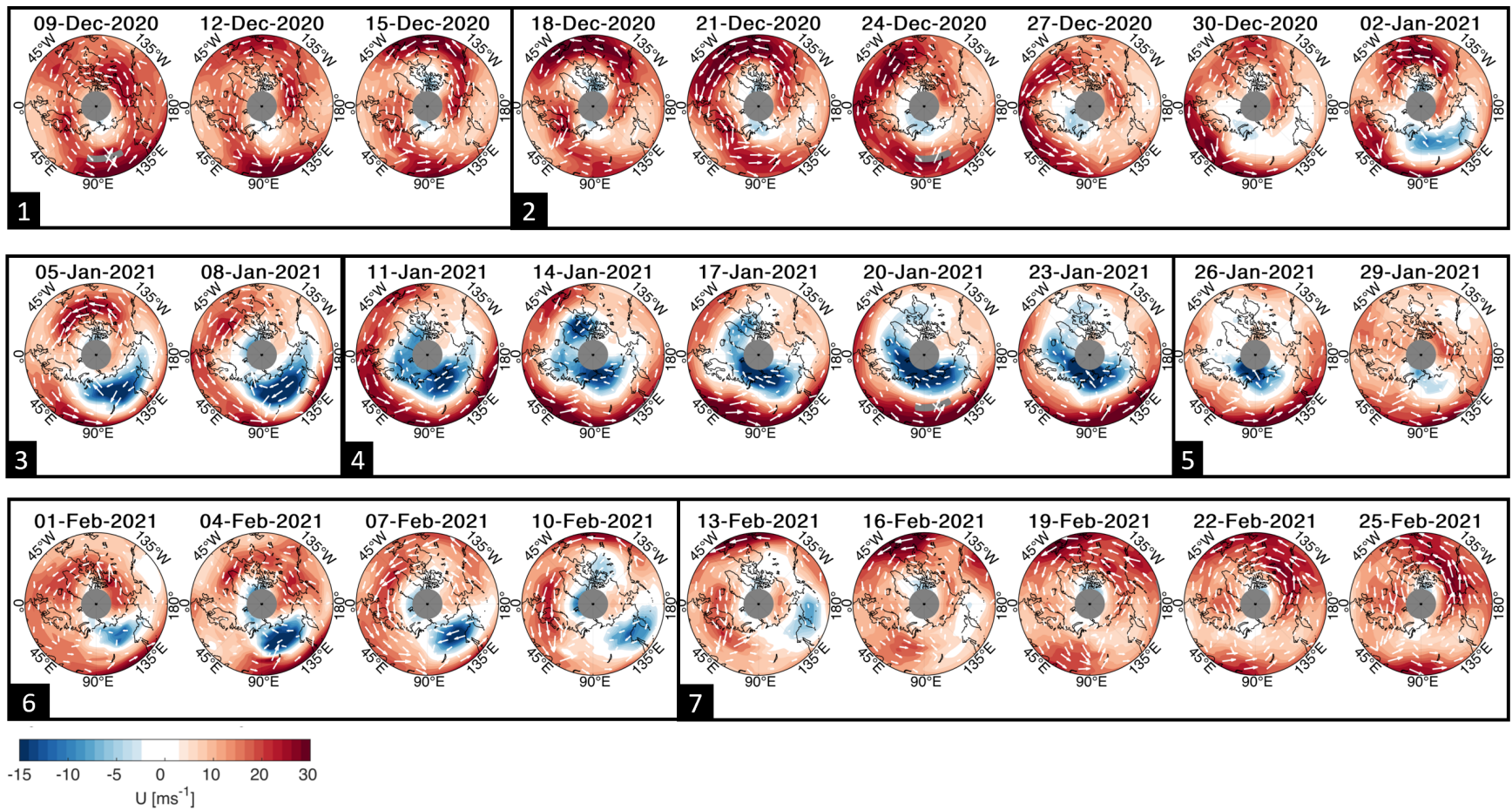

Figure 7. Five-day-mean Aeolus wind at $17 \mathrm{~km}$ altitude between $45^{\circ} \mathrm{N}$ and $80^{\circ} \mathrm{N}$. Colours show zonal wind speed; note asymmetric colour scale used to better highlight westward winds. Overlaid arrows show wind vectors, computed from $u$ and $v$; $v$ has been scaled by an empirically-chosen factor of $\times 10$. To highlight patterns, arrows are consistently sized within each panel but not between panels. Numbered boxes indicate 'phases' of the SSW, defined to help guide discussion.

same results for $u$, but with much more noise in arrow direction due to large uncertainties in $v$; one-day means do not provide full geographic coverage.

Figure 8 show three-day-mean Aeolus-derived $u$ for our seven selected dates. Equivalent plots for all dates shown in Figure 7, providing a more complete three-dimensional overview of the SSW evolution, are included as Supplementary Figure S2 but not discussed further here. The data are plotted inside a volume covering the region poleward of $60^{\circ} \mathrm{N}$ from heights of $5-22 \mathrm{~km}$, and are shown as 3D isosurfaces set at $u=+10 \mathrm{~ms}^{-1}$ (red) and $-10 \mathrm{~ms}^{-1}$ (blue). Surface topography maps are shown at the base of each volume, and are to true vertical scale with the winds; these maps extend beyond the polar regions for context, with the $60^{\circ} \mathrm{N}$ southern data limit indicated by a grey circle at surface level. A semi-transparent grey cylinder fills the region poleward of the northern data limit at $80^{\circ} \mathrm{N}$.

The SSW evolves over our seven phases as follows.

1. [Pre-SSW]: In phase 1, the polar vortex is strong and mostly circular. Maps (Figure 7) show uninterrupted and strongly positive $u$; vector winds flow in a near-zonal circle around the pole. The $u>10+\mathrm{ms}^{-1}$ surface almost completely occupies the plotted volume except at the lowest altitudes (Figure 8). Local $u$ in the vortex-edge winds reaches values 
https://doi.org/10.5194/wcd-2021-16

Preprint. Discussion started: 30 March 2021

(C) Author(s) 2021. CC BY 4.0 License.

Weather and

Climate Dynamics

Discussions

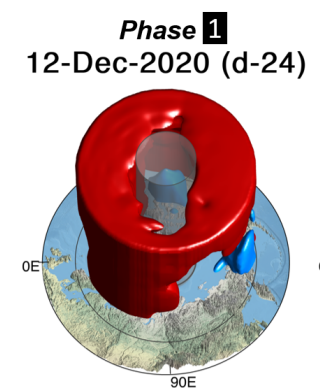

Phase 5
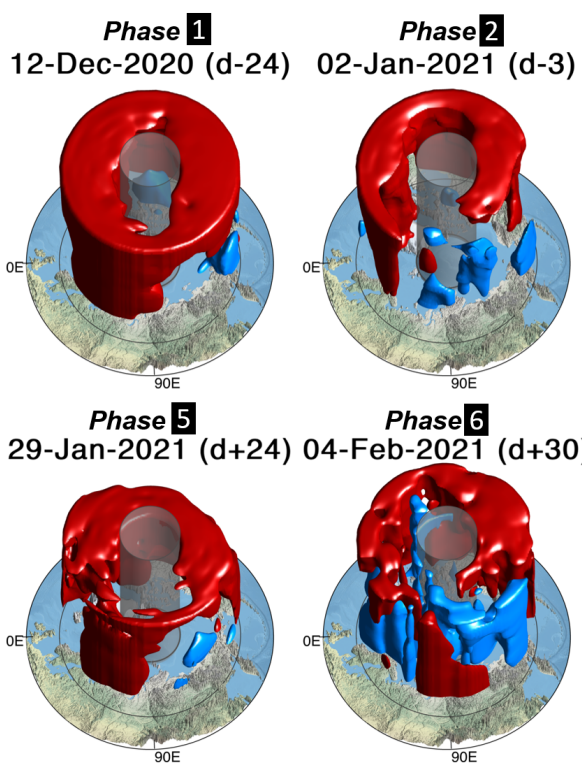

Phase 6
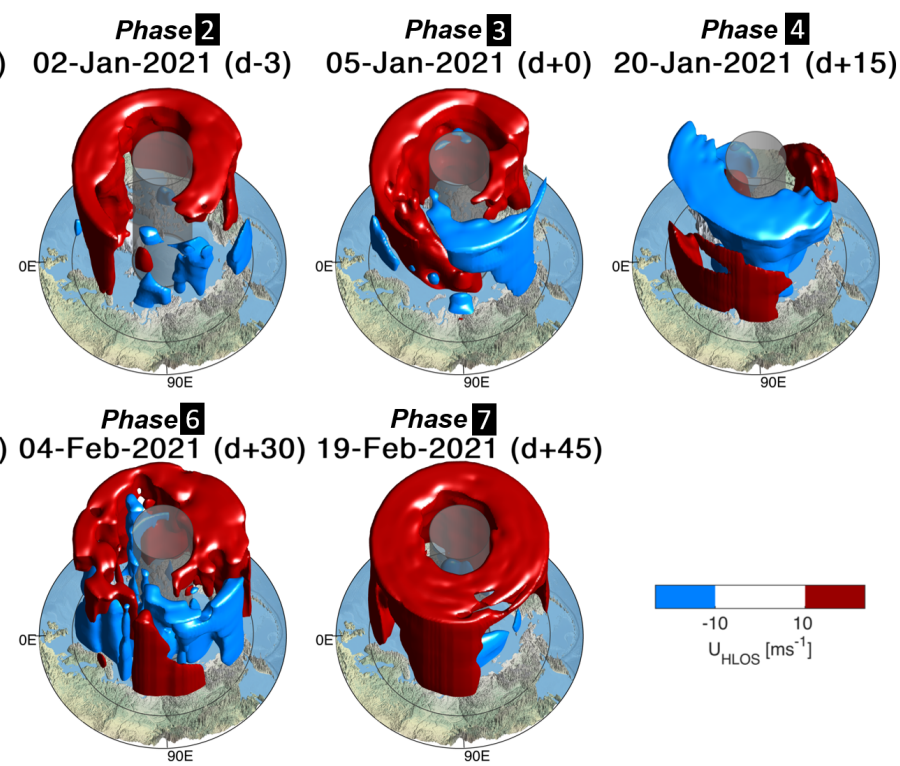

Phase 7
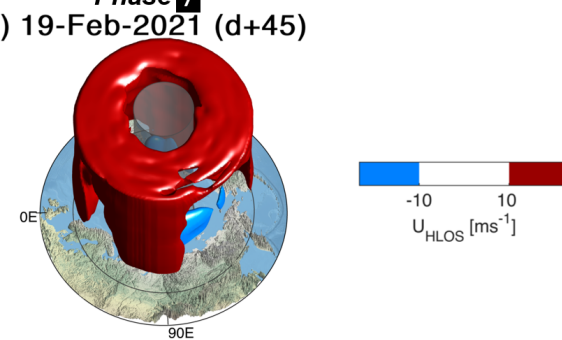

Figure 8. Three-day-mean Aeolus $u$ between $60^{\circ} \mathrm{N}$ (indicated by a line at surface level) and $80^{\circ} \mathrm{N}$ (indicated by the outer surface of the central semi-transparent grey cylinder) for selected dates during winter 2020/21. Values in brackets after each date indicate day numbers relative to the 5th of January 2021. Red (blue) surfaces enclose regions with wind speeds $>10 \mathrm{~ms}^{-1}$ in the eastward (westward) direction. Data are plotted from $5 \mathrm{~km}$ to $22 \mathrm{~km}$ altitude on a $0.75 \mathrm{~km}$ vertical grid. Terrain is shown at true relative height, using mean values on a $10 \mathrm{~km} \times 10 \mathrm{~km}$ regular spatial grid centred at the pole. Isosurfaces have been closed at the limits of the plotted volume for visual clarity, but are very likely to extend beyond it in the real atmosphere.

$u>+25 \mathrm{~ms}^{-1}$. While $u$ during this period is atypically strong relative to our climatology (Section 3 ), the observed morphology is typical for winter Aeolus data. The vortex in GPH at this time has already slipped slightly off the pole (Figure 5 and Section 7.2), but otherwise this period is broadly consistent with our theoretical understanding of the undisturbed winter atmosphere.

2. [Vortex Breakdown]: In late December and early January, the UTLS vortex begins to break down. At $17 \mathrm{~km}$, we see patches of first white and then blue appearing, corresponding to localised regions of rapidly-decelerating $u . \bar{u}$ (Figure 4 ) is still positive, but a significant fraction of the polar volume now has $u<+10 \mathrm{~ms}^{-1}$ and small regions have reached $u<-10 \mathrm{~ms}^{-1}$. There is some suggestion of a developing split into two subvortices, one centred over northern Canada and the other over the Arctic Ocean north of Russia, inferred from positive- $u$ flows over mainland Canada, negative- $u$ flows over Russia, and the direction of the vector winds near the $80^{\circ} \mathrm{N}$ data limit. Such an interpretation is consistent with our reanalysis-derived vortex metrics (Section 5) and with the morphology of the $70 \mathrm{hPa}$ GPH field (Section 7.2), both discussed further below. 
3. [Onset]: A major SSW is declared as winds at $10 \mathrm{hPa}, 60^{\circ} \mathrm{N}$ reach zero; this occurs around $10 \mathrm{~km}$ above the top of our measurement volume. By this time, a large fraction of polar UTLS $u$ has already reversed. A helical structure begins to develop in wind (Figure 8), with a volume of positive $u$ at high altitudes over North America and low altitudes over northwestern Russia underlying a volume of negative $u$ at high altitudes over Scandinavia which grows to extend to all altitudes above northeastern Russia. At the centre of the region of negative $u$, wind speeds have reached values as low as $u<-15 \mathrm{~ms}^{-1}$ at $17 \mathrm{~km}$.

4. [Peak SSW]: Here, the SSW is in full swing in the lower stratosphere. The earlier attempt at splitting the vortex appears to have failed, and instead near-uniform negative $u$ is seen in all areas poleward of $60^{\circ}$ at $17 \mathrm{~km}$ altitude with the exception of a small region over Alaska. This phase corresponds to the negative $\bar{u}$ patch seen in Figure $4 \mathrm{~b}$ at altitudes above $19 \mathrm{~km}$, and to the large positive temperature anomaly seen at these heights in Figure 4a. The helical structure seen in phase 3 remains, but with a large reduction in the volume occupied by $u>+10 \mathrm{~ms}^{-1}$ and a large increase in the volume with $u<-$ $10 \mathrm{~ms}^{-1}$. The region of $u>+10 \mathrm{~ms}^{-1}$ above North America has completely disappeared, and the region of $u<-10 \mathrm{~ms}^{-1}$ above Russia and Europe has grown significantly.

5. [Initial Recovery]: The polar vortex begins to recover in late January. We see positive $u$ at all locations at $17 \mathrm{~km}$ except for a small region over the northern Pacific. This corresponds to the local maximum in $\bar{u}$ seen in Figure 4, and represents an initial attempt by the vortex to spin back up.

6. [Second Onset]: In Figure 1, we see a secondary minimum in MLS and reanalysis $\bar{u}$ at $32 \mathrm{~km}$ at the start of this phase. Over the following days, $\bar{u}$ reduces at all heights (Figure $4 \mathrm{~b}$ ), and a negative $u$ region in Figure 7 over Russia develops again, together with a jumble of positive and negative $u$ volumes over Europe and the seas north of Great Britain. A second patch of negative wind also briefly develops over Canada towards the end of this phase in the $17 \mathrm{~km}$ maps.

7. [Final Recovery]: Finally, the vortex returns to normal. By the end of this phase, the UTLS atmospheric flow is again strong and circular around the pole, and values of $u>10+\mathrm{ms}^{-1}$ fill most of the volume. This state is very typical for polar stratosphere at this time of year (Figure 2), thus representing a return to near-normality, albeit with significant anomaly temperatures at altitudes above our wind data (Figure 3a).

\subsection{Vortex Temporal Evolution}

Figure 9 show maps of (upper half) $10 \mathrm{hPa}$ and (lower half) $70 \mathrm{hPa}$ ERA5 GPH. The data have been plotted at the seven dates previously selected in Figure 8, and an additional date one month before phase 1 has been included to show the pre-SSW form of the vortex. Empirically-selected contours intended to highlight the shape of the vortex and to help guide our discussion below are shown in red and blue. The $10 \mathrm{hPa}$ level is chosen for consistency with the general literature on SSWs and Figure 5. The $70 \mathrm{hPa}$ level is chosen as the closest model-output pressure level to the $17 \mathrm{~km}$ altitude level shown in Figure $7(70 \mathrm{hPa} \sim$ $18.5 \mathrm{~km}$; recall that our Aeolus data is binned onto a $2 \mathrm{~km}$ grid).

$17 \mathrm{~km}$ altitude Aeolus wind vectors have been overlaid on the lower panels; as previously, the $v$ component of Aeolus wind has been empirically scaled by a factor of $\times 10$. Wind vectors equatorward of $40^{\circ} \mathrm{N}$ have been omitted; this is because wind 
https://doi.org/10.5194/wcd-2021-16

Preprint. Discussion started: 30 March 2021

(c) Author(s) 2021. CC BY 4.0 License.

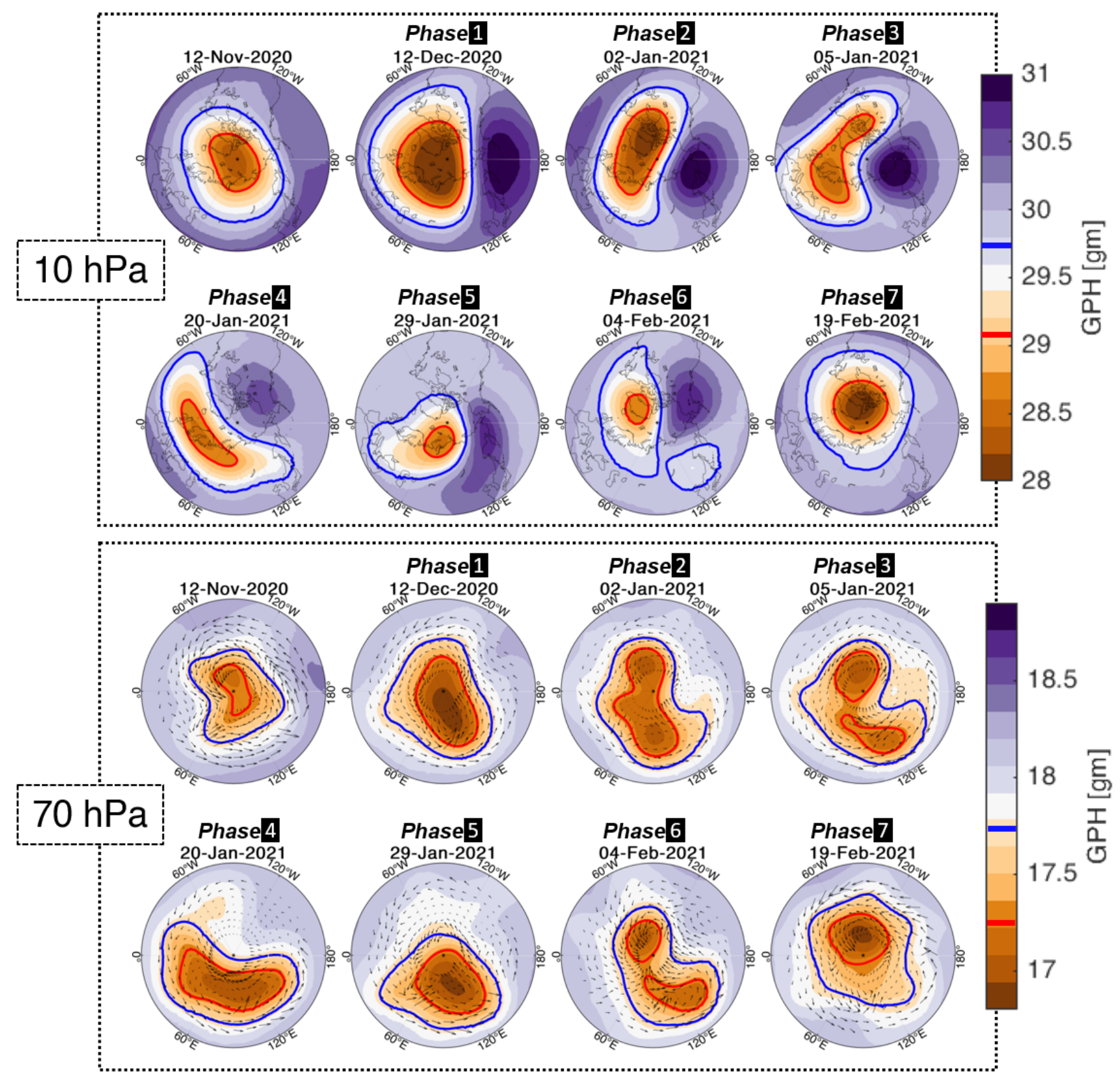

Figure 9. Maps of ERA5 (top) $10 \mathrm{hPa}$ (bottom) $70 \mathrm{hPa}$ GPH in $\mathrm{m}$ for selected days at 00:00. Selected contours have been highlighted in blue and red, with their locations indicated on the colour bars. Aeolus wind vectors at $17 \mathrm{~km}$ and poleward of $40^{\circ}$ have been overlaid on the lower panel, and the continents removed to aid visual interpretation. Wind vectors have been scaled in the $v$ direction by a factor of $\times 10$. 
https://doi.org/10.5194/wcd-2021-16

Preprint. Discussion started: 30 March 2021

(c) Author(s) 2021. CC BY 4.0 License.

(c) (i)

Weather and

Climate Dynamics

Discussions
L

speeds in the midlatitude jet are significantly faster than in many regions nearer the pole, and visually dominate the Figure

if included. These wind vectors follow the contours of the vortex-edge in GPH exceptionally closely at all timepoints shown given the assumptions implicit in our reprojection into $u$ and $v$, again demonstrating the high fidelity of Aeolus wind products.

The temporal progression of the vortex can be followed clearly through these maps and the summary lines shown in Figure 5. We divide this discussion into three distinct narrative sections, as follow.

\subsubsection{Pre-SSW, Phase 1}

515 In mid-November, the $10 \mathrm{hPa}$ vortex is roughly circular and centred very close to the pole. It begins to drift south over the next few weeks, briefly bouncing back towards the pole at the very beginning of December (Figure 5). By the 12th of December (phase 1 map) the vortex centre has clearly moved, but is still centred relatively near the pole at $78^{\circ} \mathrm{N}$, approximately on the prime meridian. This first six weeks of data correspond to the period of largely-typical stratospheric weather seen at the left-hand side of Figure 3.

At the $70 \mathrm{hPa}$ level the vortex is less regular in form, consistent with expected atmospheric dynamics at this height. It is somewhat irregular in shape in November, and has started to elongate along the $120^{\circ} \mathrm{E}$ axis by the 12 th of December. Aeolus wind vectors follow the edge of the GPH-derived vortex contours closely, including a tight detour towards the pole slightly east of the prime meridian in November.

\subsubsection{Vortex Breakdown, Onset and Peak SSW, Phases 2-4}

525 The equatorward drift of the $10 \mathrm{hPa}$ vortex core begins to accelerate at the end of December, again interrupted by a brief movement poleward (Figure 5). As January begins, the vortex elongates along an axis aligned from the Caspian Sea to Hudson Bay, briefly exceeding an aspect ratio of 2.4 (Figure 5). A few days after this, the zonal mean wind speed at $60^{\circ} \mathrm{N}$ reaches zero and the SSW becomes major. Over the next two weeks the vortex remains at its southernmost point and is highly elongated, with a negative GPH anomaly stretching from western Russia and Scandinavia over the North Atlantic and (in the early part of the period) Canada. This period represents the maximum of lower-stratospheric $\bar{T}$ and the minimum of $60^{\circ} \mathrm{N} \bar{u}$ (Figures 3 and 4), i.e. the peak of the SSW.

At $70 \mathrm{hPa}$ the vortex splits at around the $17.4 \mathrm{~g} . \mathrm{km}$ contour during phase 2, deepening to the $17.3 \mathrm{~g} . \mathrm{km}$ contour (red line) in phase 3, and exhibits separate but joined minima over Canada and central Russia, with the saddle between the minima lying over the Arctic Ocean east of Greenland. By phase 4 this bipolar structure has dissolved, leaving a clear single minimum spanning mainland Russia. This minimum is centred $45^{\circ}$ east of the $10 \mathrm{hPa} \mathrm{GPH}$ minimum over Scandinavia; consistent with the helical wind structure seen in the lower half of this altitude range in Figure 8.

Wind vectors in these snapshots continue to closely mirror the vortex edge. In phases 2 and 3, wind data suggests that either two separate circulations or a single circulation in close to the form of a figure- 8 have formed flowing anticlockwise around the two minima; unfortunately due to lack of data coverage at the very highest latitudes we do not observe the region where these circulations would meet. 
https://doi.org/10.5194/wcd-2021-16

Preprint. Discussion started: 30 March 2021

(c) Author(s) 2021. CC BY 4.0 License.

(c) (i)

Weather and

Climate Dynamics

Discussions
L

During phase 4, we again see winds closely following the form of the $70 \mathrm{hPa}$ vortex core, with eastward flows south of the region enclosed by the $17.3 \mathrm{~g} . \mathrm{km}$ contour (red line) and westward flows north of the region. Winds speeds (i.e. arrow lengths) inside the contour are significantly lower than those to the south, and slightly smaller than those to the north. Winds on the other side of the pole are both relatively (Figure 9) and absolutely (Figure 7) low in this period, contributing to the near-zero at $60^{\circ} \mathrm{N}$ during this period (Figure 4). This period corresponds to the latter part of the displacement-like period in Figure 5 .

\subsubsection{Initial Recovery, Second Onset, and Final Recovery, Phases 5-7}

In phase 5, the vortex core at $10 \mathrm{hPa}$ shifts eastwards and slightly northwards, becoming more circular and centred over the Arctic ocean poleward of north-central Russia with an extension into Eastern Europe. At the 70 Pa level, it is centred slightly southeast of the $10 \mathrm{hPa}$ centre, and also forms a single less-elliptical mass covering most of Russia. Winds are strong and zonal along the southern edge of the vortex core, and in general flow anticlockwise around the region of minimum GPH. Wind speeds are large on the south side of the vortex and small on the other side of the pole.

As we move into phase 6, we again see a brief attempt by the vortex to split at both levels; at the $10 \mathrm{hPa}$ level this occurs at the $29.8 \mathrm{~g} . \mathrm{km}$ contour (blue line), while at the $70 \mathrm{hPa}$ level we again see a saddle forming over Greenland which splits the data at the $17.3 \mathrm{~g} . \mathrm{km}$ contour (red line). This snapshot has the poorest correspondence between GPH and the wind vectors of the eight snapshots: while we again see some evidence of either two separate or one figure- 8 circulations at the $70 \mathrm{hPa}$ level around the minima in GPH, the winds in the Canadian minimum are strong and northwards and those at the northern edge of the Russian minimum are more disrupted and less clearly flowing around the minimum than in our other snapshots. This may be an artefact of time-averaging the Aeolus data; phase 6 varies rapidly in form (Figures 7-8) and while we have shown a single timepoint for model GPH, we have used a five-day averaging for our wind vectors which may not be respond quickly enough to the evolution of the geophysical situation to match the GPH contours. Such a problem would also affect phase 5, our shortest phase, but as the situation is less rapidly-varying there we do not see a mismatch.

Finally, in Phase 7, we see a return to normal winter conditions. At both the $10 \mathrm{hPa}$ and $70 \mathrm{hPa}$ levels, the vortex is centred slightly south of but close to, the pole, with minimum GPH along the $80^{\circ} \mathrm{W}$ meridian but poleward of $85 \circ$. Winds at the $70 \mathrm{hPa}$ level are maximal close to the longitude of the vortex minimum and on the mirror side of the vortex, and follow contours of GPH likely associated with a weak high-mode planetary wave (light purple).

\section{Surface Coupling and Surface Impacts}

SSWs often have significant effects on surface weather. These effects typically take place through indirect coupling processes over the weeks following the SSW, including via modulation of the jet stream, the Northern Annular Mode, and other processes which imprint upon GPH (e.g. Baldwin and Dunkerton, 2001; Kidston et al., 2015; Ming, 2015).

To investigate such coupling, Figure 10a shows normalised area-weighted polar-cap-mean GPH anomalies (hereafter $\mathrm{Z}^{\prime}$ ) averaged from $65-90^{\circ} \mathrm{N}$ derived from ERA5 data relative to a $1979-2020$ climatology. Note that this climatological period differs from our previous analyses, which use the post-2004 MLS period only. 

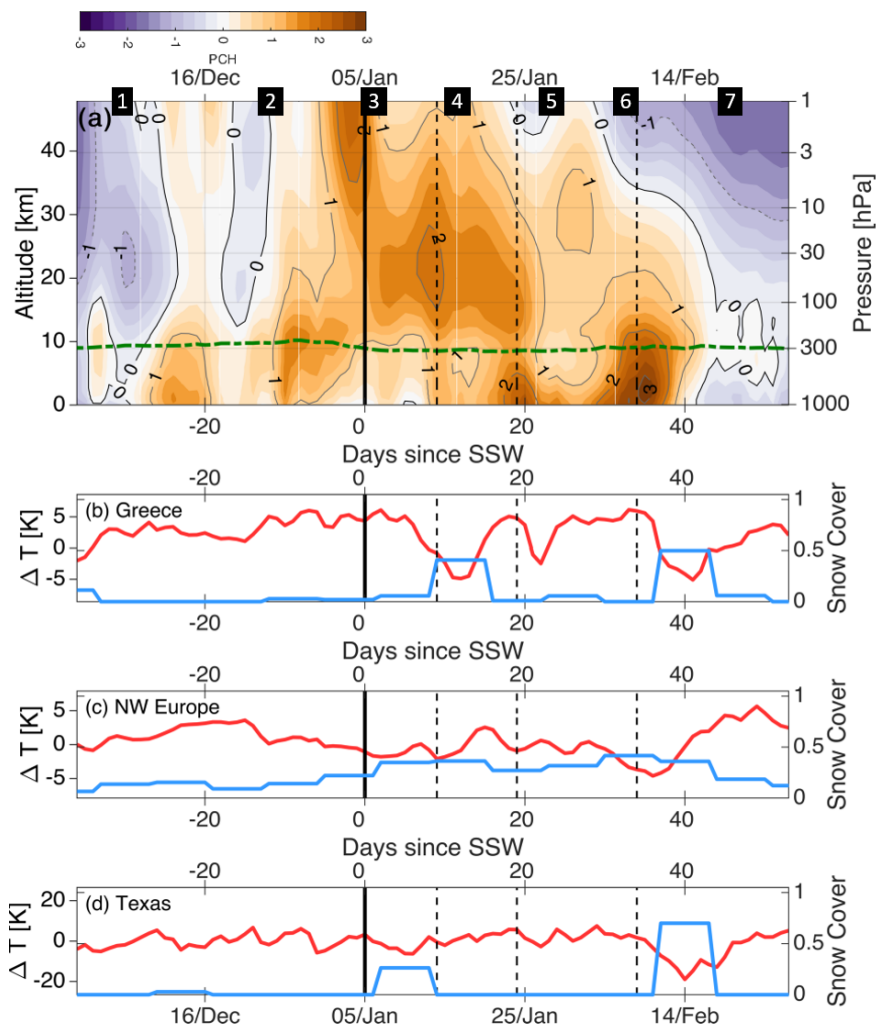

Figure 10. (a) Polar cap height anomalies $\left(Z^{\prime}\right)$ around the January 2021 SSW relative to a 1979-2020 climatology, derived from ERA5 GPH. Green dash-dotted line indicates tropopause height. (b-d) time series of (red,left axis) $2 \mathrm{~m}$ temperature anomaly and (blue) fractional snow cover extent for three selected geographic regions. For all panels, the thick solid vertical line indicated the $10 \mathrm{hPa}$ date of the SSW commencing, and the thin dashed lines corresponding to local post-SSW minima of Z at zero altitude. Numbered boxes refer to SSW phases discussed above.

We first describe the temporal evolution of $Z^{\prime}$ in the UTLS and above.

- Absolute stratospheric $Z^{\prime}$ does not exceed 1 until the last two days of December. $Z^{\prime}$ is negative at the beginning of December, turns positive in mid-December, then becomes negative again until near the end of the month. The positive $\mathrm{Z}^{\prime}$ period corresponds to the beginning of the vortex breakdown (Figures 4, 7,9), and happens at approximately the same time as minima are seen in both $\overline{u^{\prime} v^{\prime}}$ and $\overline{v^{\prime} T^{\prime}}$ at all heights (Figure 6), with the minimum at the start of the $\mathrm{Z}^{\prime}$ peak in $\overline{u^{\prime} v^{\prime}}$ and the end in $\overline{v^{\prime} T^{\prime}}$.

- From the 26th of December, $Z^{\prime}$ increases at all heights. This happens first in the UTLS, where it coincides with falling Aeolus $\bar{u}$ (Figure 4). A few days later, $Z^{\prime}$ also begins to increase above $30 \mathrm{~km}$, at the same time as MLS $\bar{u}$ rapidly reverses

(Figure 3). These separate regions of high $Z^{\prime}$ both spread vertically into the lower stratosphere with time, converging at 
https://doi.org/10.5194/wcd-2021-16

Preprint. Discussion started: 30 March 2021

(c) Author(s) 2021. CC BY 4.0 License.

\section{(c) (i)}

Weather and

Climate Dynamics

Discussions

$\sim 20 \mathrm{~km}$ altitude around the start of the SSW as the vortex centroid reaches its most southerly point and becomes highly elongated (Figures 5 and 9).

- As the SSW evolves, stratospheric $Z^{\prime}$ remains high for an extended period, with lower-stratospheric $Z^{\prime}>1.75$ for twenty days and $>0$ for over forty days after the SSW commences. In the upper stratosphere, we see $Z^{\prime}>0$ for most of this period, with a small local maximum at $\sim+25$ days corresponding again to strong local minima in $\overline{u^{\prime} v^{\prime}}$ and $\overline{v^{\prime} T^{\prime}}$ at all heights (Figure 6) and to a brief period of increased $\bar{u}$ in Figure 4.

- From the beginning of February, $Z^{\prime}$ begins to fall, crossing 0 at the beginning of February and continuing to reach -1.75 by the end of the month. This decline begins at the highest altitudes before propagating downwards, and coincides with wind speeds throughout the column returning to climatology.

We next investigate how the SSW may have coupled to and impacted upon surface weather, using $Z^{\prime}$ as a proxy metric of stratosphere-troposphere-surface coupling. To quantify surface weather effects, Figure 11 shows consecutive five-day-mean $2 \mathrm{~m}$ temperature anomaly (hereafter $2 \mathrm{mT}^{\prime}$ ) maps at the hemispheric scale from the beginning of the SSW to the end of February. Supporting our discussion, we also (Figures 10b-d) show fractional snow cover extent and $2 \mathrm{mT}^{\prime}$ averaged over Greece (specifically, $\left.20-30^{\circ} \mathrm{E}, 35-45^{\circ} \mathrm{N}\right)$, Northwestern Europe $\left(10^{\circ} \mathrm{W}-20^{\circ} \mathrm{E}, 45-65^{\circ} \mathrm{N}\right)$, and Texas $\left(105-95^{\circ} \mathrm{W}, 45-65^{\circ} \mathrm{N}\right) .2 \mathrm{mT}^{\prime}$ has been derived from ERA5 output; snow cover extent has been produced by interpolating weekly values from Robinson et al. (2014) to a daily scale. $2 \mathrm{mT}^{\prime}$ in Figures 10 and 11 is again computed relative to a 1979-2020 climatology.

We structure our discussion in terms of Figure 11, referring to Figures 10b-d to highlight some selected specific events with a strong possibility of stratospheric linkages.

From the 7th of January, we see negative $2 \mathrm{mT}^{\prime}$ over Western Europe, associated with extreme snowfall in Madrid and the surrounding area (not shown). Based on both the early date of this event relative to the SSW lifecycle and on the lack of any obvious $Z^{\prime}$ feature linking the stratospheric vortex breakdown to the surface (Figure 10a), we believe that this event was not caused by the SSW. The cooler average temperatures in Europe prior to and around the commencement of the SSW are however consistent with the work of Kolstad et al. (2010) and King et al. (2019).

By the 17th of January, $2 \mathrm{mT}^{\prime}$ surface structures characteristic of SSW surface impacts have begun to appear (e.g. Butler et al., 2017), with a cold anomaly in Siberia and a warm anomaly over Baffin Bay. This follows the development of a positive $Z^{\prime}$ link from the lower stratosphere to the surface (first dotted line from left, Figure 10). Over the days following this $Z^{\prime}$ link, we also see reduced $2 \mathrm{mT}^{\prime}$ and heavy snowfall over Greece (Figure 10b), together with a local maximum in snow cover extent and minimum in $2 \mathrm{mT}^{\prime}$ over NW Europe (Figure 10c).

From the 27 th of January, negative $2 \mathrm{mT}^{\prime}$ begins to appear over the mainland United States, and positive $2 \mathrm{mT}^{\prime}$ in the Middle East, simultaneously with a low-latitude maximum of $Z^{\prime}>2$ (Figure 10a). An unusual feature here is the development of positive $2 \mathrm{mT}^{\prime}$ over the Urals, which persists into early February and is associated with high pressure over the Urals advecting warm air from the south. This feature may have acted to inhibit westward extension of Siberian cold anomalies, and prevented northeastern Europe from developing a strong cold anomaly before this date. 

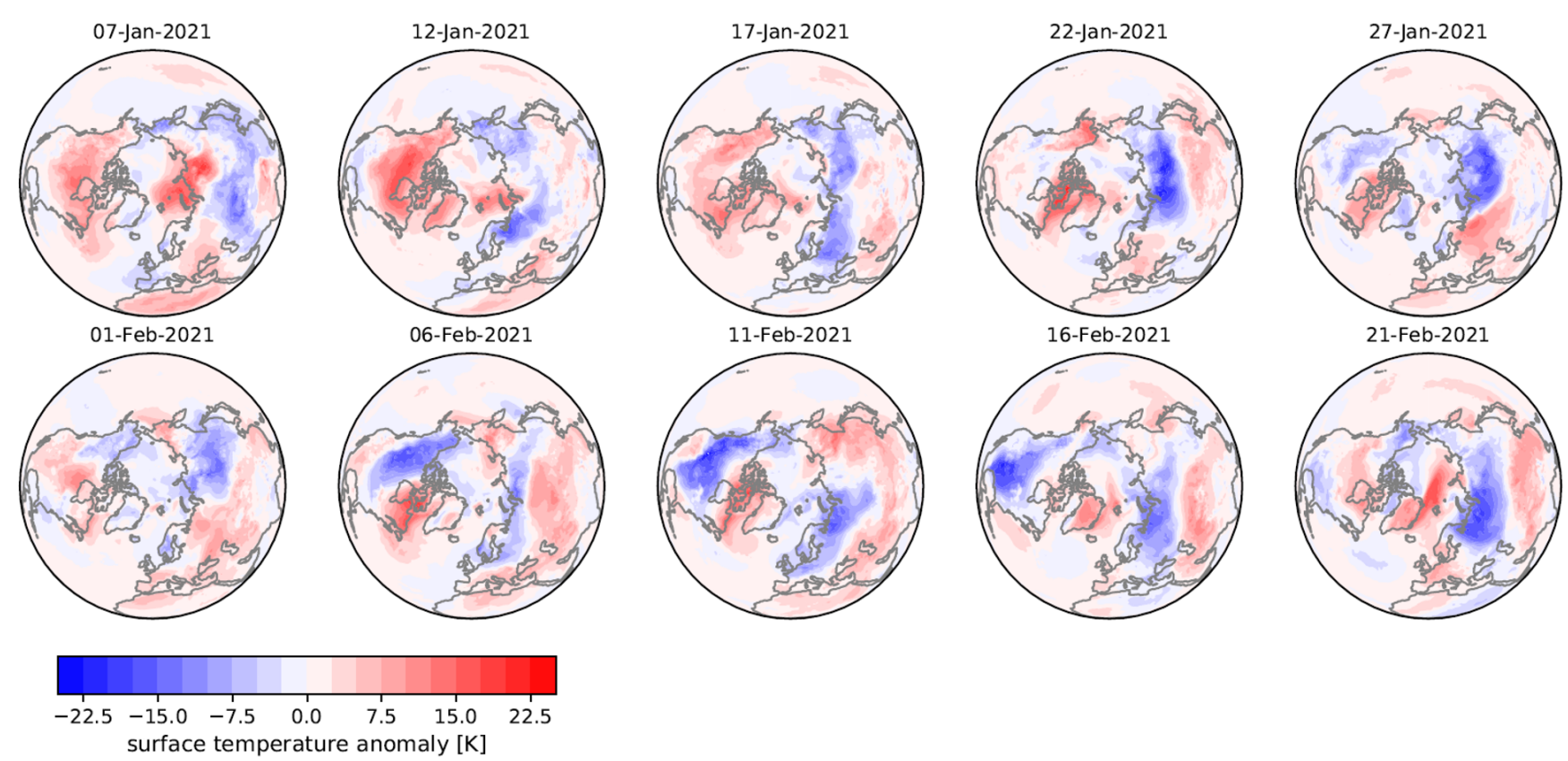

Figure 11. Consecutive five-day-mean maps of ERA5 $2 \mathrm{~m}$ temperature anomalies for the period following the SSW. Values are shown as anomalies from a 1979-2020 climatology for the given dates.

From the 1st of February, cold $2 \mathrm{mT}^{\prime}$ moves southward over North America, starting over Alaska and western Canada, then intensifying over the mainland United States and reaching Texas by 11th Feb. This US cold air outbreak was the coldest February weather in this region since 1989, and can be clearly seen in Figure 10d as a very large negative $2 \mathrm{mT}^{\prime}(\sim-20 \mathrm{~K}$ relative to climatology) with high snow coverage, which commences synchronously with a strong positive $\mathrm{Z}^{\prime}>3$ and in turn suggests a strong possibility of a role for stratosphere-troposphere coupling. The cold outbreak was intensified by increased high pressure over the Aleutian region which acted to block the jet stream, causing a southward loop of the jet downstream of the block and advecting cold air southwards. The increased Aleutian high here may have been in part due to pre-existing La Niña conditions in the tropical Pacific during this winter; furthermore, increased blocking over the Canadian Archipelago may have acted to push cold further south than usual.

Northwestern Europe experiences its most intense cold spell from the 1st to the 11th of February, also synchronously with this large $\mathrm{Z}^{\prime}$ anomaly. A local-minimum temperature was reached at Braemar on the 11th of February, which at $250 \mathrm{~K}$ was the lowest UK temperature since 1995. This cold air shifts eastwards by the 16th of February, delaying the development of cold snowy weather in Greece relative to NW Europe, and leading to Athens experiencing $20-25 \mathrm{~cm}$ of snow on the 15 th and 16 th of February.

Although data of this type cannot show a direct causal link, our data therefore strongly suggest that the early January SSW may have acted as either a trigger or an intensifier for several extreme winter weather events affecting densely-populated 
https://doi.org/10.5194/wcd-2021-16

Preprint. Discussion started: 30 March 2021

(c) Author(s) 2021. CC BY 4.0 License.

(c) (i)

Weather and

Climate Dynamics

Discussions
L.

regions of the Northern Hemisphere over the next two months. Even if the SSW did play an important role in these extreme events, our analysis is also not able to explain why different regions may have been impacted at different times during the SSW evolution; such an investigation is left to future studies.

\section{Discussion and Conclusions}

In the Introduction, we outlined two key objectives for this study, which were to (1) characterise SSW-induced dynamical changes for this event using novel observational data and (2) to assess the suitability of Aeolus data for studying future extreme weather events of this type. Accordingly, we divide our discussions and conclusions between these two headings.

\subsection{Objective 1: Dynamical Changes and Impacts}

1. Unlike many others, this SSW was a mixed event not easily classifiable as having either a split or a displacement vortex. Considering for example the criteria of Seviour et al. (2013), the vortex was displaced for long enough but not far enough south to be a displacement event, with an aspect ratio elliptical enough but for too brief a period to be a split event.

2. At a bulk climatological level, the SSW was fairly typical, with zonal mean temperatures and winds in the normal range for such an event (Section 2.4). However, closer study identifies atypical features, including a second zero-crossing of $60^{\circ} 10 \mathrm{hPa}$ wind nearly a month after commencement associated with a recovering and then re-collapsing vortex (Section 7), and highly anomalous mesoscale eddy forcing during and after the event (Section 6)

3. Aeolus wind data strongly correlate spatially with GPH estimates from ERA5, supporting their accuracy. The GPH estimates, in turn, show evidence of downward-coupling supporting the hypothesis that several major extreme-weather events during January and February 2021, including cold and snow cover extent extrema in Greece, Northwestern Europe and, especially, Texas were likely related to some degree to the SSW at the beginning of the year. This demonstrates the large and significant impact of SSWs on surface climate, and highlights the importance of improving our stratospheric forecasting capabilities.

\subsection{Objective 2: Assessments of Aeolus Data}

1. Aeolus data are suitable for scientific use in dynamically-extreme events such as SSWs. Even using inherently-limited estimates of $u$ and $v$ reprojected from 1D data and (in the case of $v$ ) only approximately scaled for magnitude, Aeolus wind agrees well with both MLS and ERA5 (Section 2.4), supports direct estimation of mesoscale fluxes consistent with reanalysis (Section 6), produces detailed and internally-consistent 2D and 3D wind structure (Section 7.1, and shows excellent agreement at a physical level with the evolution of the vortex in ERA5 GPH (Section 7.2).

2. Aeolus data contain fine vertical structures. A key example of this are the helical wind features seen in Figure 8 during phases 3, 4 and 6, which represent structures only a few kilometres in vertical extent even after applying heavy vertical $(2 \mathrm{~km})$, horizontal $\left(5^{\circ} \times 20^{\circ}\right)$ and temporal (three-day) averaging to ensure full coverage. Although we do not do so here, 
https://doi.org/10.5194/wcd-2021-16

Preprint. Discussion started: 30 March 2021

(C) Author(s) 2021. CC BY 4.0 License.
Weather and

Climate Dynamics

Discussions

exploitation of the Rayleigh data at its true spatiotemporal resolution and of the even finer-resolution Mie data could provide additional useful information on possible filamentary wind structures related to the vortex breakdown of the SSW.

3. Aeolus consistently measures much higher zonal-mean wind maxima in the bursts of positive zonal wind seen in the UTLS before the SSW (Figure 4, Appendix B). This suggests that ERA5 has difficulty reproducing short bursts of high winds relative to observations.

4. Aeolus winds may contain features not present in ERA5 output, although these differences could well be due to Aeolus measurement errors (including due to day-to-day changes in spatial sampling) or errors induced by our reprojection, and more detailed study will be required to confirm if they are real. Two main examples of this are seen in this study, (a) a period of internally-consistent sign difference from reanalysis $\overline{v^{\prime} T^{\prime}}$ (Figure 6a) and (b) a several-day differences in timing the timing of when zonal-mean $\bar{u}$ reached zero at the top of the Aeolus column (Figure 1); the minimum $\bar{u}$ reached in observations is also deeper and longer-lasting. In the later case, MLS geostrophic $u$ provides supplementary evidence of this being an error in the reanalysis.

Code and data availability. Aeolus data can be obtained from the ESA Aeolus web portal, https://aeolus.services/. MLS data can be obtained from the NASA DISC, https://disc.gsfc.nasa.gov/. ERA5 data can be obtained from the Copernicus Climate Data Store https://cds.climate.copernicus.eu/, and ERA5T data from ECMWF's MARS service. Snow extent data were obtained from Robinson et al. (2014). All of these data are freely available, with the exception of ERA5T data on model levels - at time of submission these require member-state access to the ECMWF API, but will be replaced by freely-available ERA5 data (expected to be identical) within three months of analysis date. The code used to produce the analyses and figures in this study has been archived at doi:10.5281/zenodo.4638273.

Author contributions. CJW developed the concept of the study, carried out most of the data analyses presented, wrote the initial draft of the manuscript, and produced all figures except Figures 11 and A1. RJH carried out the vortex-moment analyses for Figure 5, the surfacecoupling analysis for Figure 10, and produced and analysed the data for Figure 11. TPB acquired and preprocessed the Aeolus data used, and carried out vital work underpinning the data analysis pipeline. NPH produced Figure A1, and the associated text. WJS suggested calculating heat and momentum fluxes, and assisted significantly in interpreting these results. All authors contributed to understanding and interpreting the data and to finalising the manuscript text.

Competing interests. The authors have no competing interests. 
https://doi.org/10.5194/wcd-2021-16

Preprint. Discussion started: 30 March 2021

(c) Author(s) 2021. CC BY 4.0 License.

(c) (1)

Weather and

Climate Dynamics

Discussions

Acknowledgements. CJW, DMM, NPH and RJH are funded by NERC grant NE/S00985X/1. CJW is also funded by Royal Society University

Research Fellowship UF160545, and DMM by NERC Independent Research Fellowship NE/N014057/1. TPB is funded by EPSRC grant EP/R513155/1 and by Royal Society grant RGF/EA/180217. 
https://doi.org/10.5194/wcd-2021-16

Preprint. Discussion started: 30 March 2021

(c) Author(s) 2021. CC BY 4.0 License.

(c) (i)

Weather and

Climate Dynamics

Discussions

\section{Appendix A: Projecting HLOS Wind into the Zonal and Meridional Directions}

Aeolus measures the projection of the horizontal zonal and meridional wind vectors $u$ and $v$ in the HLOS direction. In this study, we project the measured HLOS wind into zonal and meridional components to obtain $u_{\mathrm{HLOS}}$ and $v_{\mathrm{HLOS}}$.

Figure A1 illustrates the geometry of this process, where $\theta$ is the azimuth of the HLOS direction measured clockwise from north. The Aeolus measured HLOS wind is the sum of projections from both the zonal and meridional winds as

695 HLOS wind $=u \sin \theta+v \cos \theta$

Our projected winds $u_{\text {HLOS }}$ and $v_{\text {HLOS }}$ are then given by

$u_{\mathrm{HLOS}}=u \sin ^{2} \theta+v \sin \theta \cos \theta$

$v_{\mathrm{HLOS}}=u \sin \theta \cos \theta+v \cos ^{2} \theta$

We can see from the above that $u_{\text {HLOS }}$ and $v_{\text {HLOS }}$ are not independent, and it is possible for $u_{\text {HLOS }}$ to be contaminated by projections of the meridional wind $v$ and vice versa. However, since tropospheric $u$ is typically larger than $v$, and the angle $\theta$ is greater than 70 degrees equatorward of $70^{\circ} \mathrm{N}$, we can estimate that this effect is likely to be small for $u_{\mathrm{HLOS}}$.

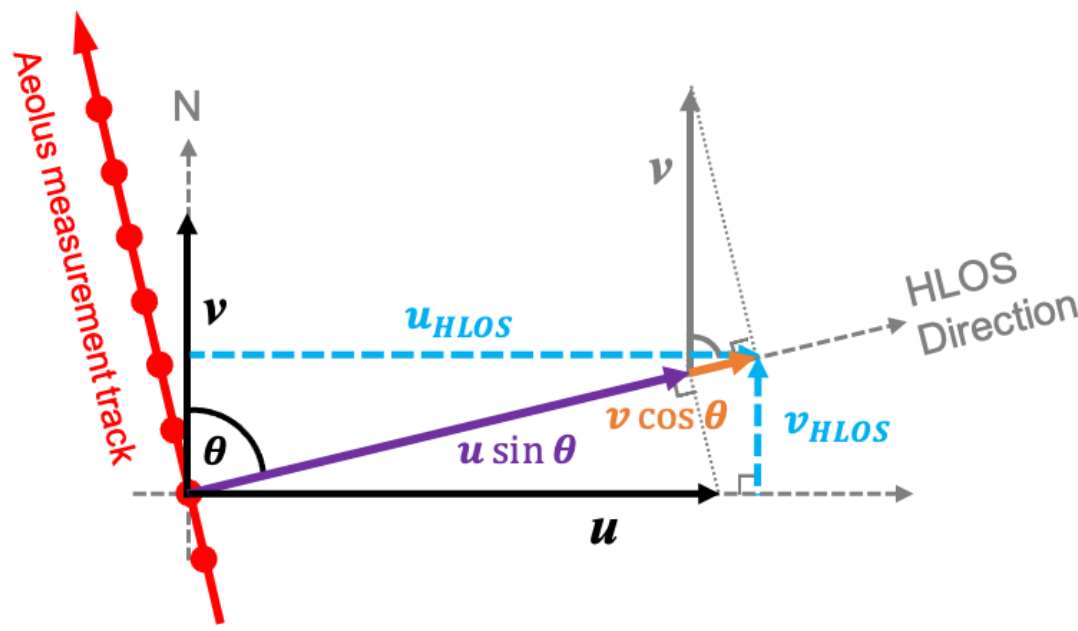

Figure A1. Schematic diagram showing the Aelous horizontal line of sight (HLOS) viewing geometry, where $\theta$ is the angle between the HLOS direction and north. When Aeolus makes wind measurements, the zonal and meridional wind vectors $u$ and $v$ are projected into the HLOS direction. This measured HLOS wind then projected into $u_{\text {HLOS }}$ and $v_{\text {HLOS }}$ shown throughout the study. 
https://doi.org/10.5194/wcd-2021-16

Preprint. Discussion started: 30 March 2021

(c) Author(s) 2021. CC BY 4.0 License.

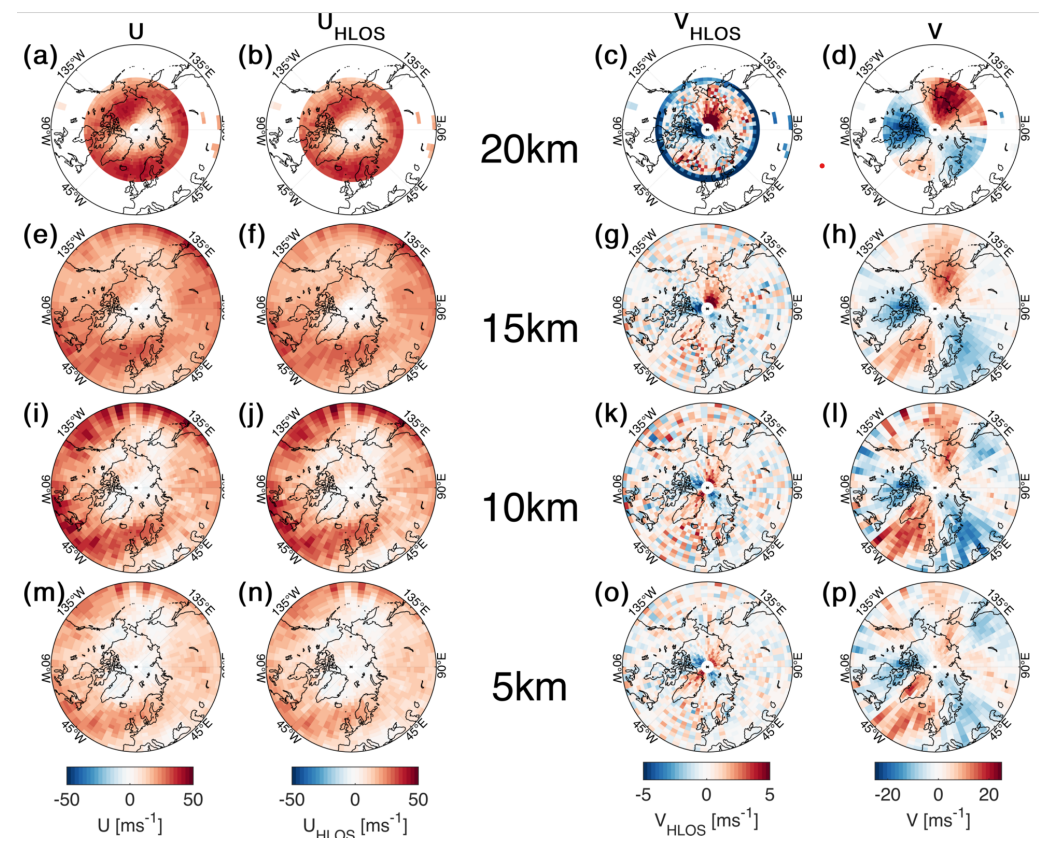

Figure A2. Aeolus-sampled ERA5 estimates of $u, v, u_{\mathrm{HLOS}}$ and $v_{\mathrm{HLOS}}$. (a,e,i,m) show $u,(\mathrm{~b}, \mathrm{f}, \mathrm{j}, \mathrm{n}) u_{\mathrm{HLOS}},(\mathrm{c}, \mathrm{g}, \mathrm{k}, \mathrm{o}) v_{\mathrm{HLOS}}$ and (d,h,l,p) $v$ at (a-d) $20 \mathrm{~km},(\mathrm{e}-\mathrm{h}) 15 \mathrm{~km},(\mathrm{i}-1) 10 \mathrm{~km}$ and (m-p) $5 \mathrm{~km}$.

To further constrain any errors due to this ambiguity, we have assessed the differences between $u$ and $u_{\mathrm{HLOS}}$ and between $v$ and $v_{\text {HLOS }}$ for ERA5 reanalysis winds. To do this, we first sample $u$ and $v$ from ERA5 at the times and locations of all Aeolus measurements in January 2020, then project these into the satellite-observation frame of reference to estimate the HLOS wind, then project this back into cardinal directions to estimate $u_{\mathrm{HLOS}}$ and $v_{\mathrm{HLOS}}$.

Figure A2 shows these sampled fields at four height levels. Sampled $u_{\mathrm{HLOS}}$ (second column from left) corresponds well in terms of both morphology and magnitude with the input $u$ field (first column). Sampled $v_{\text {HLOS }}$ shows similar morphology to the input $v$ field, but with a much smaller magnitude: $v_{\mathrm{HLOS}}$ (third column) is typically a factor $\sim 10 \times$ smaller than the input $v$ fields (fourth column) at any given point.

Figures A3(a,e,i,m) and (d,h,l,p) show the same data as probability distributions of pointwise differences ERA5 $u-u_{\mathrm{HLOS}}$ and $v-v_{\text {HLOS }}$ respectively, as a function of latitude at four height levels. In each panel, five grey lines are shown, indicating (from top to bottom) the 2.5th, 18th, 50th (median), 82nd and 97.5th percentiles of the pointwise distribution. These percentiles are chosen as they represent the non-parametric equivalents of the first and standard deviations of a normally-distributed dataset.

For $u-u_{\text {HLOS }}$, differences are small near the Equator at all altitudes, increasing with latitude. This is consistent with Aeolus' orbital geometry, which will have a meridional LOS at the polar turnaround latitudes and a near-zonal LOS at the Equator. Accordingly, the width of the distribution increases dramatically at the poles, with the $2.5-97.5 \%$ range reaching a width of $\pm 20 \mathrm{~ms}^{-1}$ at high altitudes. Importantly, however, the majority of the measurements remains tightly distributed around the zero-difference line (black-dashed), with the median diverging only by low single-digit values at all heights, and 
https://doi.org/10.5194/wcd-2021-16

Preprint. Discussion started: 30 March 2021

(c) Author(s) 2021. CC BY 4.0 License.

Weather and

Climate Dynamics

Discussions

L.
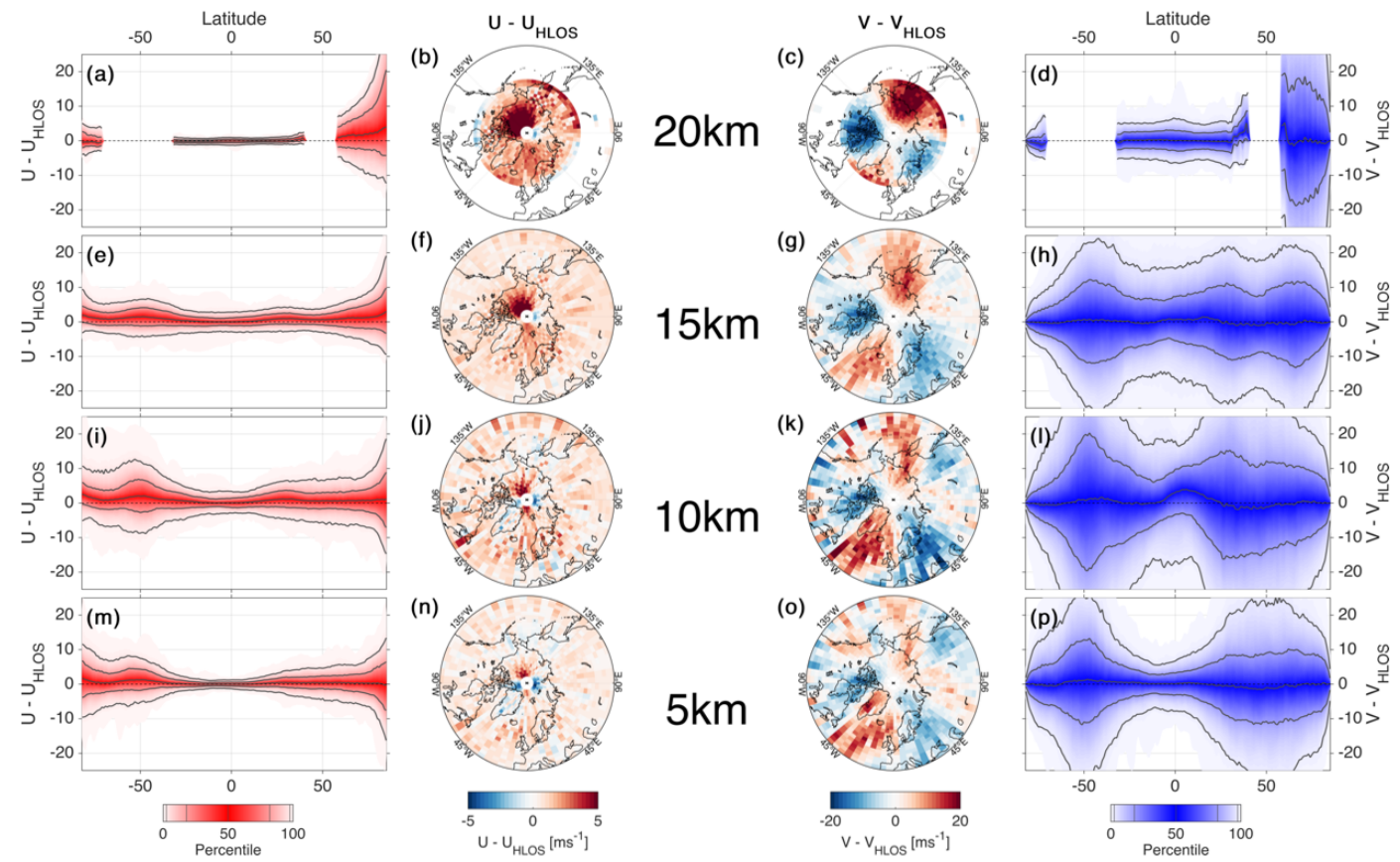

Figure A3. Estimated error $u-u_{\text {HLOS }}$ due to projection from HLOS to direction-resolved wind, computed using the Aeolus January 2020 sampling pattern and ERA5 reanalysis winds. (a,d,e,h,i,l,m,p) probability distribution of pointwise error as a function of latitude for (a,e,i,m) zonal (d,h,l,p) meridional winds. (b,c,f,g,j,k,n,o) maps of mean error for (b,f,j,n) zonal (c,g,k,o) meridional winds.

the $18-82 \%$ range reaching widths of at most $10 \mathrm{~ms}^{-1}$. There is a positive skew to the distribution, indicating a tendency to overestimate the true zonal wind using this technique in this region; 2019/20 was a record-strong year for the polar vortex from late January onwards (Lawrence et al., 2020), and thus the equivalent errors for our 2020/21 study may be more evenly split by sign than in this test case.

Reprojected meridional winds perform much worse than zonal at most latitudes, with an $18-82 \%$ range approaching $>20 \mathrm{~ms}^{-1}$ at most latitudes. This is consistent with the very large magnitude differences seen in Figure A2.

Figures A3(b,c,f,g,j,k,n,o) map the mean differences in the (b,f,j,n) zonal and (c,g,k,o) meridional directions, again at four height levels. In the zonal direction, these mean differences are typically $<3 \mathrm{~ms}^{-1}$ at most locations and heights, except at the $20 \mathrm{~km}$ altitude level where they routinely exceed $5 \mathrm{~ms}^{-1}$. A systematic positive skew is seen in most cases, consistent with the probability distribution.

Meridional geographic mean differences are much larger and evenly split between positive and negative differences, with values approaching and sometimes exceeding $20 \mathrm{~ms}^{-1}$ at all heights. The meridional pattern seen corresponds extremely closely in both spatial pattern and amplitude to an underlying pattern in meridional wind seen in ERA5 output during this period (Figure A2, rightmost column); this is because removing the very small-magnitude $v_{\text {HLOS }}$ magnitudes from the original $v$ fields makes only a marginal difference to the final results. 

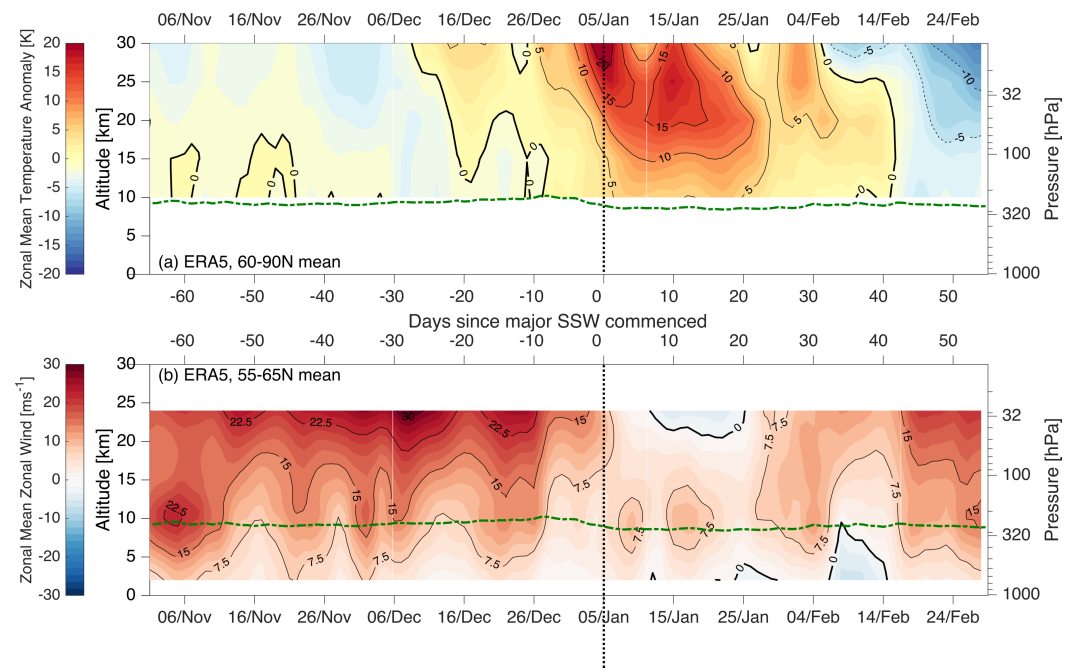

Figure B1. ERA5-derived (a) $60^{\circ} \mathrm{N}-90^{\circ} \mathrm{N}$ mean temperature anomalies; (b) $55^{\circ} \mathrm{N}-65^{\circ} \mathrm{N}$ mean zonal winds for winter $2020 / 21$, for altitudes 0-30 km. Mean tropopause height is shown as a dot-dashed green line. Vertical dotted line indicates the date at which $10 \mathrm{hPa}$ winds reversed and the SSW became defined as major.

Based on this analysis, we conclude that the use of projected zonal winds is in general safe for our analysis provided not too much scientific weight is placed on individual point-values; that is to say that the data are largely reliable when averaged across several individual points. Projected meridional winds, however, are much less robust, and in particular cannot be safely used for individual-point analysis. They do however have a broadly correct morphology at large lengthscales, and therefore can be of scientific use provided they are averaged over large areas and account is taken that wind magnitude will be very heavily suppressed. Although we do not investigate it directly here, $v_{\mathrm{HLOS}}$ estimates may be of more scientific use in the tropics, where the spread range narrows markedly; this is consistent with the observational geometry of the satellite, as the line-of-sight vector will be directed in a near-meridional direction near the Equator.

\section{Appendix B: Differences between ERA5 and Observations in the Zonal Mean}

Figure B1 shows zonal-mean ERA5 (a) temperature and (b) winds, in the same graphical form as MLS and Aeolus data in Figure 4. Figure B2 shows the same data as a difference from the equivalent observations. The data used in Figure B2 have been smoothed by three times before differencing, as daily differences exhibit significant day-to-day noise which we assume to be due to satellite spatial sampling.

At a broad level, the data are highly similar in the zonal mean, with deviations not exceeding $3 \mathrm{~K} / 4 \mathrm{~ms}^{-1}$ except for a small number of poorly-sampled bins at the very top of the Aeolus column. In general, ERA5 reproduces MLS temperature well with differences falling approximately evenly to either side of zero. Differences are also spread around zero for wind, but with a clear bias towards higher wind speeds in Aeolus. 

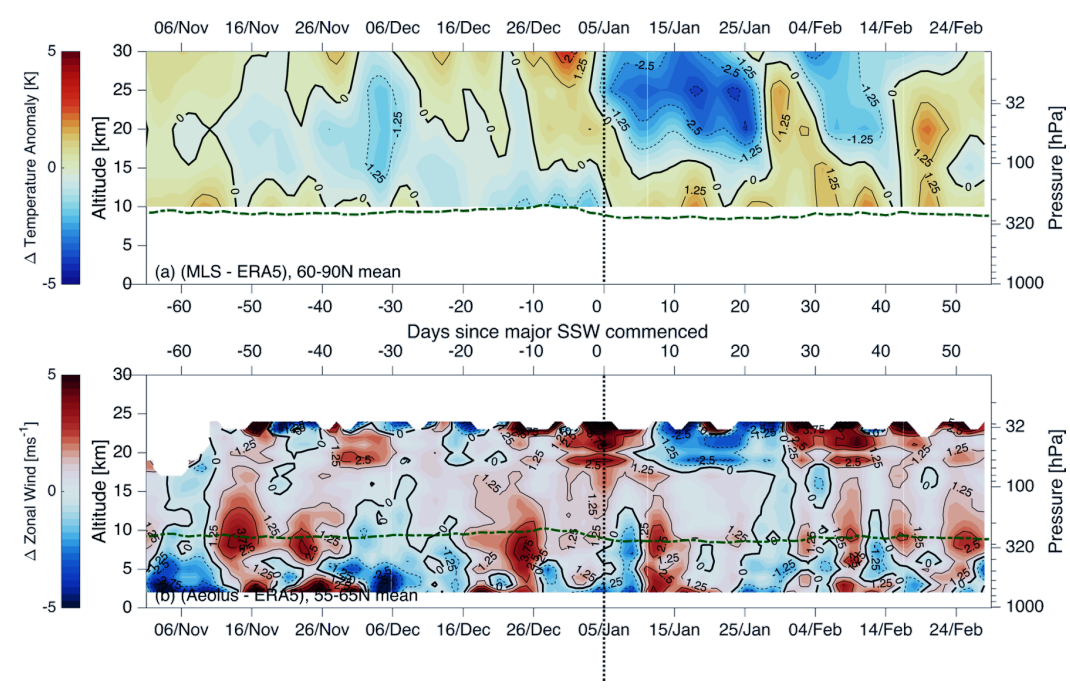

Figure B2. Difference between Figures B1 and 4. Data have been smoothed three days in time to compensate for satellite sampling patterns.

ERA5 $\bar{T}$ and $\bar{U}$ are significantly smoother than the observational data, consistent with their origin in an assimilative model. The period of only small temperature anomalies and timing of the repeated speeding up and slowing down of the vortex-edge winds in November and early December are well-reproduced by the reanalysis, but the maxima of wind speed in this bursts are significantly underestimated by the reanalysis, with these maxima representing the largest differences outside the top binning level.

The extreme temperature variations of the SSW are well reproduced by ERA5, despite the physical complexity of the event, with differences remaining below $3 \mathrm{~K}$. After the SSW, a long-lasting high-bias is seen in ERA5 relative to MLS covering most of the lower stratosphere (i.e. a negative difference MLS-ERA5), lasting until winds begin to accelerate again at the end of January. The reduction and reversal of zonal winds at the top of the Aeolus column is much shallower in both depth and magnitude in ERA5, and the region of enhanced wind around the tropopause shortly after the SSW is split into two distinct regions. The speeding up and slowing down of the lower-stratospheric vortex after the SSW is well-reproduced in ERA5, as is the final restoration in late February, again with a notable low-bias in ERA5's ability to produce short-term wind maxima. 
https://doi.org/10.5194/wcd-2021-16

Preprint. Discussion started: 30 March 2021

(C) Author(s) 2021. CC BY 4.0 License.
Weather and

Climate Dynamics

Discussions

\section{References}

Baldwin, M. P. and Dunkerton, T. J.: Stratospheric Harbingers of Anomalous Weather Regimes, Science, 294, 581-584, https://doi.org/10.1126/science.1063315, https://doi.org/10.1126/science.1063315, 2001.

Banyard, T. P., Wright, C. J., Hindley, N. P., Halloran, G., Krisch, I., Kaifler, B., and Hoffmann, L.: Atmospheric Gravity Waves in Aeolus Wind Lidar Observations, https://doi.org/10.1002/essoar.10506308.1, https://doi.org/10.1002/essoar.10506308.1, 2021.

Butler, A. H., Sjoberg, J. P., Seidel, D. J., and Rosenlof, K. H.: A sudden stratospheric warming compendium, Earth System Science Data, 9, 63-76, https://doi.org/10.5194/essd-9-63-2017, https://doi.org/10.5194/essd-9-63-2017, 2017.

Chanin, M., Garnier, A., Hauchecorne, A., and Porteneuve, J.: A Doppler lidar for measuring winds in the middle atmosphere., Geophysical research letters, 16, https://doi.org/10.1029/GL016i011p01273, 1989.

Charlton, A. J. and Polvani, L. M.: A New Look at Stratospheric Sudden Warmings. Part I: Climatology and Modeling Benchmarks, Journal of Climate, 20, 449-469, https://doi.org/10.1175/jcli3996.1, https://doi.org/10.1175/jcli3996.1, 2007.

Charlton-Perez, A. J., Huang, W. T. K., and Lee, S. H.: Impact of sudden stratospheric warmings on United Kingdom mortality, Atmospheric Science Letters, https://doi.org/10.1002/asl.1013, https://doi.org/10.1002/asl.1013, 2020.

Copernicus Climate Change Service (C3S), .: ERA5: Fifth generation of ECMWF atmospheric reanalyses of the global climate, https: //cds.climate.copernicus.eu/cdsapp\#!/home.

ESA: ALADIN - Atmospheric Laser Doppleer Instrument. Working Group Report, ESA SP-1112, p. 45p, 1989.

ESA: ADM-Aeolus Science Report, ESA SP-1311, p. 121p, 2008.

France, J. A., Harvey, V. L., Randall, C. E., Hitchman, M. H., and Schwartz, M. J.: A climatology of stratopause temperature and height in the polar vortex and anticyclones, Journal of Geophysical Research: Atmospheres, 117, n/a-n/a, https://doi.org/10.1029/2011jd016893, https://doi.org/10.1029/2011jd016893, 2012.

Gerber, E. P., Martineau, P., Ayarzagüena, B., Barriopedro, D., Bracegirdle, T. J., Butler, A. H., Calvo, N., Hardiman, S. C., Hitchcock, P., Iza, M., Langematz, U., Lu, H., Marshall, G., Orr, A., Palmeiro, F. M., Son, S.-W., and Taguchi, M.: Chapter 6: Extratropical Stratosphere-troposphere Coupling (submitted), Tech. rep., Stratospheric Reanalysis Intercomparison Report, 2021.

Hall, R. J., Mitchell, D. M., Seviour, W. J., and Wright, C. J.: Tracking the stratosphere-to-surface impact of Sudden Stratospheric Warmings, Journal of Geophysical Research: Atmospheres, https://doi.org/10.1029/2020jd033881, https://doi.org/10.1029/2020jd033881, 2020.

Hersbach, H., Bell, B., Berrisford, P., Hirahara, S., Horányi, A., Muñoz-Sabater, J., Nicolas, J., Peubey, C., Radu, R., Schepers, D., Simmons, A., Soci, C., Abdalla, S., Abellan, X., Balsamo, G., Bechtold, P., Biavati, G., Bidlot, J., Bonavita, M., Chiara, G., Dahlgren, P., Dee, D., Diamantakis, M., Dragani, R., Flemming, J., Forbes, R., Fuentes, M., Geer, A., Haimberger, L., Healy, S., Hogan, R. J., Hólm, E., Janisková, M., Keeley, S., Laloyaux, P., Lopez, P., Lupu, C., Radnoti, G., Rosnay, P., Rozum, I., Vamborg, F., Villaume, S., and Thépaut, J.N.: The ERA5 global reanalysis, Quarterly Journal of the Royal Meteorological Society, 146, 1999-2049, https://doi.org/10.1002/qj.3803, https://doi.org/10.1002/qj.3803, 2020.

Kidston, J., Scaife, A. A., Hardiman, S. C., Mitchell, D. M., Butchart, N., Baldwin, M. P., and Gray, L. J.: Stratospheric influence on tropospheric jet streams, storm tracks and surface weather, Nature Geoscience, 8, 433-440, https://doi.org/10.1038/ngeo2424, https://doi. org/10.1038/ngeo2424, 2015.

King, A. D., Butler, A. H., Jucker, M., Earl, N. O., and Rudeva, I.: Observed Relationships Between Sudden Stratospheric Warmings and European Climate Extremes, Journal of Geophysical Research: Atmospheres, 124, 13 943-13 961, https://doi.org/10.1029/2019jd030480, https://doi.org/10.1029/2019jd030480, 2019. 
https://doi.org/10.5194/wcd-2021-16

Preprint. Discussion started: 30 March 2021

(C) Author(s) 2021. CC BY 4.0 License.
Weather and

Climate Dynamics

Discussions

Kolstad, E. W., Breiteig, T., and Scaife, A. A.: The association between stratospheric weak polar vortex events and cold air outbreaks in the Northern Hemisphere, Quarterly Journal of the Royal Meteorological Society, 136, 886-893, https://doi.org/10.1002/qj.620, https: //doi.org/10.1002/qj.620, 2010.

Kretschmer, M., Cohen, J., Matthias, V., Runge, J., and Coumou, D.: The different stratospheric influence on cold-extremes in Eurasia and North America, npj Climate and Atmospheric Science, 1, https://doi.org/10.1038/s41612-018-0054-4, https://doi.org/10.1038/ s41612-018-0054-4, 2018.

Lawrence, Z. D., Perlwitz, J., Butler, A. H., Manney, G. L., Newman, P. A., Lee, S. H., and Nash, E. R.: The Remarkably Strong Arctic Stratospheric Polar Vortex of Winter 2020: Links to Record-Breaking Arctic Oscillation and Ozone Loss, Journal of Geophysical Research: Atmospheres, 125, https://doi.org/10.1029/2020jd033271, https://doi.org/10.1029/2020jd033271, 2020.

Livesey, N., Snyder, W. V., Read, W., and Wagner, P.: Retrieval algorithms for the EOS Microwave limb sounder (MLS), IEEE Transactions on Geoscience and Remote Sensing, 44, 1144-1155, https://doi.org/10.1109/tgrs.2006.872327, https://doi.org/10.1109/tgrs.2006.872327, 2006.

Livesey, N. J., Read, W. G., Wagner, P. A., Froidevaux, L., Lambert, A., Manney, G. L., Millán Valle, L., Pumphrey, H. C., Santee, M. L., Schwartz, M. J., Wang, S., Fuller, R. A., Jarnot, R. F., Knosp, B. W., and Martinez, E.: Earth Observing System (EOS) Aura Microwave Limb Sounder (MLS) Data Quality and Description, version 4.2, 2015.

Manney, G. L., Krüger, K., Pawson, S., Minschwaner, K., Schwartz, M. J., Daffer, W. H., Livesey, N. J., Mlynczak, M. G., Remsberg, E. E., Russell, J. M., and Waters, J. W.: The evolution of the stratopause during the 2006 major warming: Satellite data and assimilated meteorological analyses, Journal of Geophysical Research, 113, https://doi.org/10.1029/2007jd009097, https://doi.org/10.1029/2007jd009097, 2008.

Manney, G. L., Schwartz, M. J., Krüger, K., Santee, M. L., Pawson, S., Lee, J. N., Daffer, W. H., Fuller, R. A., and Livesey, N. J.: Aura Microwave Limb Sounder observations of dynamics and transport during the record-breaking 2009 Arctic stratospheric major warming, Geophysical Research Letters, 36, https://doi.org/10.1029/2009g1038586, https://doi.org/10.1029/2009g1038586, 2009.

Manney, G. L., Lawrence, Z. D., Santee, M. L., Read, W. G., Livesey, N. J., Lambert, A., Froidevaux, L., Pumphrey, H. C., and Schwartz, M. J.: A minor sudden stratospheric warming with a major impact: Transport and polar processing in the 2014/2015 Arctic winter, Geophysical Research Letters, 42, 7808-7816, https://doi.org/10.1002/2015gl065864, https://doi.org/10.1002/2015gl065864, 2015.

Martin, A., Weissmann, M., Reitebuch, O., Rennie, M., Geiß, A., and Cress, A.: Validation of Aeolus winds using radiosonde observations and numerical weather prediction model equivalents, Atmospheric Measurement Techniques, 14, 2167-2183, https://doi.org/10.5194/amt14-2167-2021, https://doi.org/10.5194/amt-14-2167-2021, 2021.

Ming, A.: Stratosphere-troposphere coupling in the Earth system: Where next?, Weather, 70, 232-233, https://doi.org/10.1002/wea.2517, https://doi.org/10.1002/wea.2517, 2015.

Mitchell, D. M., Gray, L. J., Anstey, J., Baldwin, M. P., and Charlton-Perez, A. J.: The Influence of Stratospheric Vortex Displacements and Splits on Surface Climate, J. Clim., 26, 2668-2682, https://doi.org/10.1175/JCLI-D-12-00030.1, http://journals.ametsoc.org/doi/abs/10. 1175/JCLI-D-12-00030.1, 2013.

Pedatella, N., Chau, J., Schmidt, H., Goncharenko, L., Stolle, C., Hocke, K., Harvey, V., Funke, B., and Siddiqui, T.: How Sudden Stratospheric Warming Affects the Whole Atmosphere, Eos, 99, https://doi.org/10.1029/2018eo092441, https://doi.org/10.1029/2018eo092441, 2018. 
https://doi.org/10.5194/wcd-2021-16

Preprint. Discussion started: 30 March 2021

(C) Author(s) 2021. CC BY 4.0 License.
Weather and

Climate Dynamics

Discussions

Polvani, L. M. and Waugh, D. W.: Upward Wave Activity Flux as a Precursor to Extreme Stratospheric Events and Subsequent Anomalous Surface Weather Regimes, Journal of Climate, 17, 3548-3554, https://doi.org/10.1175/1520-0442(2004)017<3548:uwafaa>2.0.co;2, https://doi.org/10.1175/1520-0442(2004)017<3548:uwafaa>2.0.co;2, 2004.

Reitebuch, O.: The spaceborne wind lidar mission ADM-Aeolus., Atmospheric Physics, pp. 815-827, https://doi.org/10.1007/978-3-64230183-4_49, 2012.

Rennie, M. and Isaksen, L.: The NWP impact of Aeolus Level-2B Winds at ECMWF, https://doi.org/10.21957/ALIFT7MHR, https://www. ecmwf.int/node/19538, 2020.

Robinson, D. A., Estilow, T. W., and NOAA Climate Data Record Program: NOAA Climate Data Record (CDR) of Northern Hemisphere (NH) Snow Cover Extent (SCE), Version 1, https://doi.org/10.7289/V5N014G9, https://data.nodc.noaa.gov/cgi-bin/iso?id=gov.noaa.ncdc: C00756, 2014.

Safieddine, S., Bouillon, M., Paracho, A.-C., Jumelet, J., Tencé, F., Pazmino, A., Goutail, F., Wespes, C., Bekki, S., Boynard, A., HadjiLazaro, J., Coheur, P.-F., Hurtmans, D., and Clerbaux, C.: Antarctic Ozone Enhancement During the 2019 Sudden Stratospheric Warming Event, Geophysical Research Letters, 47, https://doi.org/10.1029/2020g1087810, https://doi.org/10.1029/2020g1087810, 2020.

Schoeberl, M., Douglass, A., Hilsenrath, E., Bhartia, P., Beer, R., Waters, J., Gunson, M., Froidevaux, L., Gille, J., Barnett, J., Levelt, P., and DeCola, P.: Overview of the EOS aura mission, IEEE Transactions on Geoscience and Remote Sensing, 44, 1066-1074, https://doi.org/10.1109/tgrs.2005.861950, https://doi.org/10.1109/tgrs.2005.861950, 2006.

Scott, R. K.: A new class of vacillations of the stratospheric polar vortex, Quarterly Journal of the Royal Meteorological Society, 142, 1948-1957, https://doi.org/10.1002/qj.2788, https://doi.org/10.1002/qj.2788, 2016.

Seviour, W. J. M., Mitchell, D. M., and Gray, L. J.: A practical method to identify displaced and split stratospheric polar vortex events, Geophysical Research Letters, 40, 5268-5273, https://doi.org/10.1002/grl.50927, https://doi.org/10.1002/grl.50927, 2013.

Siskind, D. E., Eckermann, S. D., Coy, L., McCormack, J. P., and Randall, C. E.: On recent interannual variability of the Arctic winter mesosphere: Implications for tracer descent, Geophysical Research Letters, 34, https://doi.org/10.1029/2007gl029293, https://doi.org/10. 1029/2007g1029293, 2007.

Stoffelen, A., Pailleux, J., Källén, E., Vaughan, J., Isaksen, L., Flamant, P., Wergen, W., Andersson, E., Schyberg, H., Culoma, A., and Meynart, R.: The atmospheric dynamics mission for global wind field measurement., Bulletin of the American Meteorological Society, 86, https://doi.org/10.1175/BAMS-86-1-73, 2005.

Tao, M., Konopka, P., Ploeger, F., Grooß, J.-U., Müller, R., Volk, C. M., Walker, K. A., and Riese, M.: Impact of the 2009 major sudden stratospheric warming on the composition of the stratosphere, Atmospheric Chemistry and Physics, 15, 8695-8715, https://doi.org/10.5194/acp15-8695-2015, https://doi.org/10.5194/acp-15-8695-2015, 2015.

Waters, J., Froidevaux, L., Harwood, R., Jarnot, R., Pickett, H., Read, W., Siegel, P., Cofield, R., Filipiak, M., Flower, D., Holden, J., Lau, G., Livesey, N., Manney, G., Pumphrey, H., Santee, M., Wu, D., Cuddy, D., Lay, R., Loo, M., Perun, V., Schwartz, M., Stek, P., Thurstans, R., Boyles, M., Chandra, K., Chavez, M., Chen, G.-S., Chudasama, B., Dodge, R., Fuller, R., Girard, M., Jiang, J., Jiang, Y., Knosp, B., LaBelle, R., Lam, J., Lee, K., Miller, D., Oswald, J., Patel, N., Pukala, D., Quintero, O., Scaff, D., Snyder, W. V., Tope, M., Wagner, P., and Walch, M.: The Earth observing system microwave limb sounder (EOS MLS) on the aura Satellite, IEEE Transactions on Geoscience and Remote Sensing, 44, 1075-1092, https://doi.org/10.1109/tgrs.2006.873771, https://doi.org/10.1109/tgrs.2006.873771, 2006.

Wright, C.: Detection of stratospheric gravity waves using HIRDLS data, Ph.D. thesis, University of Oxford, 2010.

Wright, C. J. and Banyard, T. P.: Multidecadal Measurements of UTLS Gravity Waves Derived From Commercial Flight Data, Journal of Geophysical Research: Atmospheres, 125, https://doi.org/10.1029/2020jd033445, https://doi.org/10.1029/2020jd033445, 2020. 
https://doi.org/10.5194/wcd-2021-16

Preprint. Discussion started: 30 March 2021

(c) Author(s) 2021. CC BY 4.0 License.

(c) (1)

Weather and

Climate Dynamics

Discussions

Wright, C. J. and Hindley, N. P.: How well do stratospheric reanalyses reproduce high-resolution satellite temperature measure-

875 ments?, Atmospheric Chemistry and Physics, 18, 13 703-13 731, https://doi.org/10.5194/acp-18-13703-2018, https://doi.org/10.5194/ acp-18-13703-2018, 2018.

Wright, C. J., Osprey, S. M., Barnett, J. J., Gray, L. J., and Gille, J. C.: High Resolution Dynamics Limb Sounder measurements of gravity wave activity in the 2006 Arctic stratosphere, Journal of Geophysical Research, 115, https://doi.org/10.1029/2009jd011858, https://doi. org/10.1029/2009jd011858, 2010.

880 Wright, C. J., Hindley, N. P., Moss, A. C., and Mitchell, N. J.: Multi-instrument gravity-wave measurements over Tierra del Fuego and the Drake Passage - Part 1: Potential energies and vertical wavelengths from AIRS, COSMIC, HIRDLS, MLS-Aura, SAAMER, SABER and radiosondes, Atmospheric Measurement Techniques, 9, 877-908, https://doi.org/10.5194/amt-9-877-2016, https://doi.org/10.5194/ amt-9-877-2016, 2016. 